\title{
Research Article \\ Effects of Common Factors on Dynamics of Stocks Traded by Investors with Limited Information Capacity
}

\author{
Songtao Wu, Jianmin He, and Chao Wang \\ School of Economics and Management, Southeast University, Nanjing 211189, China \\ Correspondence should be addressed to Jianmin He; hejianminseu138@126.com
}

Received 4 June 2017; Revised 25 July 2017; Accepted 7 August 2017; Published 28 September 2017

Academic Editor: Ricardo López-Ruiz

Copyright (C) 2017 Songtao Wu et al. This is an open access article distributed under the Creative Commons Attribution License, which permits unrestricted use, distribution, and reproduction in any medium, provided the original work is properly cited.

\begin{abstract}
An artificial stock market with agent-based model is built to investigate effects of different information characteristics of common factors on the dynamics stock returns. Investors with limited information capacity update their beliefs based on the information they have obtained and processed and optimize portfolios based on beliefs. We find that with changing of concerned information characteristics the uncertainty of stock price returns rises and is higher than the uncertainty of intrinsic value returns. However, this increase is constrained by the limited information capacity of investors. At the same time, we also find that dependence between returns of stock prices also increased with the changing information environment. The uncertainty and dependency pertaining to prices show a positive relationship. However, the positive relationship is weakened when taking into account the features of intrinsic values, based on which prices are generated.
\end{abstract}

\section{Introduction}

The concept of "information" is cornerstone of the EMH (Efficiency Market Hypothesis) [1, 2], which lays basis for development of other modern finance theories. Since information is an abstract concept, a large body of literature focuses on effects of information-bearing events on dynamics of the stock market [3-5]. Findings of these papers contradict the EMH and indicate that information in the stock market cannot be impounded into prices properly and immediately.

Ultimately, effects of information on stock dynamics or information efficiency of the market shall be fulfilled via activities of individual traders. For example, [6] argues that the proportion of informed traders affects information dissemination efficiency of the market. Before incorporating information into their activities or trading strategies, traders have to search and process information first. Suffering from limited information capacity, however, they cannot process and understand all information existing in the market [7]. Early studies concerning limited information capacity of decision makers are papers of Sims $[8,9]$; then its implication is investigated in the field of stock market [10-12]. They find that limited information capacity of traders may cause excessive comovement [10], underdiversification [11], and home bias [12].
Factor analysis is one of the most popular practices for both financial practitioner and researchers, such as famous three-factor model $[13,14]$ and its numerous variants. It is also an information capacity-demanding task including analyzing lots of information, such as news, industry reports, and financial reports. Among all risk factors, common risk factors are a category of special ones. Not only dynamics of stocks but also their relations can be explained by common factors [15]. In addition, common factors are more specific than the market factor to be analyzed and have more extensive application potentiality than firm-specific factors. Thus, they are extensively exploited, like common economic factors [16], common company characteristics [17], common technical factors [18], and so forth. In fact, this emphasis or passion on common factors is supported by category learning behavior of traders due to limited information capacity [10].

Previous studies about common factors are almost empirical ones, and main purposes of these researches are identifying common factors with explanatory power for dynamics of stocks, such as momentum factor [19], short-term reversal factor [20], long-term reversal factor [3], and liquidity factor [21]. However, subject to sources of sample data, certain factors only apply to certain market scenarios. This has limited 
general explanation of roles played by common factors. Analytical approaches concerning limited information capacity [10-12] are usually accompanied with rigorous assumptions to keep the model tractable. The assumptions make analytical approaches not suitable for modeling adaptive behavior of traders in a complex market inundated with all kinds of information about risk factors.

In addition, stock market is a constantly evolving system. If we attempt to incorporate this market feature into our model, it is even harder to use traditional empirical researches or analytical approaches. Hopefully, the ABM (agent-based model) in finance field or ACF (Agent-based Computational Finance), pioneered by Sante Fe Institute, shows potentiality to cope with complex information environment of the market and the adaptive information searching and learning behavior of traders $[22,23]$.

The importance of studying effects of common factors on dynamics of stocks traded by investors with limited information capacity can be understood from perspective of $\mathrm{EMH}$ and behavioral finance and from practice of risk management and portfolio construction. Given limitation of empirical and analytical approaches above, we construct an artificial stock market using ABM to shed light on the effects. The rest of the paper is organized as follows. In Section 2, we introduce the structure of stock return, microstructure of the market, and the ABM. Simulation results and discussions are presented in Section 3. Robustness check is made in Section 4. In Section 5, conclusion remark is made.

\section{The Model}

2.1. Assets and Composition of Fundamental Return. Assuming that there are $m$ stocks in the market, their fundamental returns consisted of three types of risk factors: the market factor $r_{a}$, common factors $r_{s_{j}}(j=1,2, \ldots, n)$, and each stock's firm-specific factor $r_{g_{i}}(i=1,2, \ldots, m)$. Thus, the fundamental return of $i$ th stock at period $t$ is expressed as

$$
r_{i, t}^{f}=r_{a, t}+\sum_{j=1}^{n} \beta_{i j} r_{s_{j}, t}+r_{g_{i}, t} .
$$

In (1), $r_{a, t}, r_{s_{j}, t}$, and $r_{g_{i}, t}$ are drawn from mutually independent normal distributions $N\left(\mu_{a, t}, \sigma_{a, t}^{2}\right), N\left(\mu_{s_{j}, t}, \sigma_{s_{j}, t}^{2}\right)$, and $N\left(\mu_{g_{i}, t}, \sigma_{g_{i}, t}^{2}\right)$ at each period $t[10]$. The loading coefficient $\beta_{i j}$ indicates the effect of the $j$ th common factor on the $i$ th stock; in the present we set $\beta_{i j}=1(i=1,2, \ldots, m ; j=1,2, \ldots, n)$. With given initial value of the $i$ th stock $p_{i, 0}^{f}$, intrinsic values of the stock follow the dynamic $p_{i, t+1}^{f}=p_{i, t}^{f} \exp \left(r_{i, t}^{f}\right)(t=$ $0,1, \ldots, T)$. Thus, the variance of the $i$ th stock and the covariance of the $i$ th and $k$ th stocks $(i, k=1,2, \ldots, m, i \neq k)$ are given in

$$
\begin{aligned}
\operatorname{Var}\left(r_{i}\right) & =\sigma_{a}^{2}+\sigma_{g_{i}}^{2}+\sum_{j=1}^{n} \sigma_{s_{j}}^{2}, \\
\operatorname{Cov}\left(r_{i}, r_{k}\right) & =\sigma_{a}^{2}+\sum_{j=1}^{n} \sigma_{s_{j}}^{2} .
\end{aligned}
$$

In order to simulate the dynamic characteristics of the real stock market, we assume that the mean and variance of aforementioned normal distributions are, respectively, redrawn from uniform distributions $U\left[\mu_{a, \min }, \mu_{a, \max }\right]$, $U\left[\sigma_{a, \min }^{2}, \sigma_{a, \max }^{2}\right], \quad U\left[\mu_{s, \min }, \mu_{s, \text { max }}\right], \quad U\left[\sigma_{s, \text { min }}^{2}, \sigma_{s, \text { max }}^{2}\right], \quad$ and $U\left[\mu_{g, \min }, \mu_{g, \max }\right], U\left[\sigma_{g, \text { min }}^{2}, \sigma_{g, \text { max }}^{2}\right]$ at probabilities of $\alpha_{a}, \alpha_{s}$, and $\alpha_{g}$, which indicate intensities of dynamics of risk factors.

2.2. Portfolio Optimization. There are $N$ investors with time window length $l \sim U[100,200]$ in the market. They are endowed with a certain amount of initial wealth consisting of stocks and cash; that is, $W_{0}=\sum_{i=1}^{m} q_{i, 0} \widetilde{p}_{i, 0}+C_{0}$ in which $q_{i, 0}$ and $\widetilde{p}_{i, 0}$ are initial holding shares and price of $i$ th stock. In order to balance buying and selling power, the initial holding cash is set based on the initial holding shares and prices, such that $C_{0}=\sum_{i=1}^{m} q_{i, 0} \widetilde{p}_{i, 0}$.

At the trading day $t$, a typical investor $h(h=1,2, \ldots, N)$ either participates in portfolio trading of $m$ stocks at probability $1 / l^{h}$ or holds current position waiting for better opportunities. The principle of investor $h$ for optimizing portfolio is maximizing the utility of wealth at trading day $t+l^{h}$, namely, the statistical average timing at which investor $h$ participates in trading again. According to $[24,25]$, the investor $h$ 's wealth at the trading day $t+l^{h}$ is jointly determined by the wealth at trading day $t$ and the return of portfolio over the time interval $\left[t, t+l^{h}\right]$. Therefore, the evolution of wealth is given as

$$
W_{t+l^{h}}=W_{t}+\mathbf{Z}_{t}^{h T} \mathbf{R}_{t+l^{h}}
$$

where $\mathbf{Z}_{t}^{h T}$ is the column vector of wealth where investor $h$ invests into each stock at trading day $t$, and $T$ is the operator of transpose; $\mathbf{R}_{t+l^{h}}=\left(r_{1, t+l^{h}}, r_{2, t+l^{h}}, \ldots, r_{m, t+l^{h}}\right)^{T}$ is the corresponding return vector of stocks over the interval $\left[t, t+l^{h}\right]$. Thus, investor $h$ 's objective of portfolio optimization is calculating the optimal $\mathrm{Z}_{t}^{h T}$ by maximizing utility of wealth $u\left(W_{t+l^{h}}\right)=-\exp \left(-a^{h} W_{t+l^{h}}\right)$.

According to the widely used utility function of CARA (Constant Absolute Risk Aversion) [22, 2629], the optimization goal can be formally expressed as $\max _{\mathbf{Z}_{t}^{h}} E_{t}^{h}\left[-\exp \left(-a^{h} W_{t+l^{h}}\right)\right]$, where $a^{h}>0$ is the risk aversion attitude of investor $h$. Under the assumption of normality of the expected return $\widehat{\mathbf{R}}_{t+l^{h}}=E_{t}^{h}\left(\mathbf{R}_{t+l^{h}}\right)$ on trading day $t+l^{h}$ based on information gathered at trading day $t$, it is well known that this optimization goal is equivalent to the following expression $[30,31]$ :

$$
\max _{\mathbf{Z}_{t}^{h}}\left\{W_{t+l^{h}}+\mathbf{Z}_{t}^{h T} \widehat{\mathbf{R}}_{t+l^{h}}-\frac{1}{2} a^{h} \mathbf{Z}_{t}^{h T} \widehat{\mathbf{V}}_{t+l^{h}} \mathbf{Z}_{t}^{h}\right\} .
$$

In the expression, $\widehat{\mathbf{V}}_{t+l^{h}}=\operatorname{Cov}_{t}^{h}\left(\widehat{r}_{i, t+l^{h}}, \widehat{r}_{k, t+l^{h}}\right)(i, k=$ $1,2, \ldots, m)$ is variance-covariance matrix of expected returns over the interval $\left[t, t+l^{h}\right]$. Following [32], the elements of $\widehat{\mathbf{R}}_{t+l^{h}}$ can be written as $\widehat{r}_{i, t+l^{h}}=\widehat{p}_{i, t+l^{h}} / p_{i, o}^{h}-1 \simeq \ln \widehat{p}_{i, t+l^{h}}-$ $\ln p_{i, o}^{h}(i=1,2, \ldots, m)$, where $\widehat{p}_{i, t+l^{h}}$ and $p_{i, o}^{h}$ are, respectively, the expected price and quote of the order, namely, the price at which investor $h$ hoped her orders had been traded. 
Therefore, according to the first-order partial derivative of expression (4) against $\mathbf{Z}_{t}^{h}$, we obtain the optimal portfolio given in

$$
\mathbf{Z}_{t}^{h}=\frac{1}{a^{h}}\left(\widehat{\mathbf{V}}_{t+l^{h}}\right)^{-1} \widehat{\mathbf{R}}_{t+l^{h}}
$$

2.3. Heterogeneous Agents. From (5), we know that the optimal portfolio of investor $h$ is determined by three components: the risk aversion coefficient $a^{h}$, the expected returns $\widehat{\mathbf{R}}_{t+l^{h}}$, and the variance-covariance matrix $\widehat{\mathbf{V}}_{t+l^{h}}$ of expected returns. Investors are heterogeneous for all components. In respect of risk aversion coefficient, it is randomly and independently drawn from the uniform distribution $U[0.01,0.1]$ for all investors.

Inspired by two distinctive beliefs held by investors in the real-world stock market, the present paper follows [32] and assumes that $\widehat{\mathbf{R}}_{t+l^{h}}$ and $\widehat{\mathbf{V}}_{t+l^{h}}$ are formed based on two same weighted beliefs: fundamentalist's belief $h=F$ and chartist's belief $h=C$. Therefore, $\widehat{\mathbf{R}}_{t+l^{h}}=0.5 \widehat{\mathbf{R}}_{t+l^{h}}^{F}+0.5 \widehat{\mathbf{R}}_{t+l^{h}}^{C}$ and $\widehat{\mathbf{V}}_{t+l^{h}}=$ $0.5 \widehat{\mathbf{V}}_{t+l^{h}}^{F}+0.5 \widehat{\mathbf{V}}_{t+l^{h}}^{C}$. The superscript $h$ is substituted with $F$ and $C$ to indicate different beliefs, based on which expectations are formed. From analysis in previous subsections, we know that $\widehat{\mathbf{R}}_{t+l^{h}}$ is determined by $\widehat{p}_{t+l^{h}}$ and $p_{o}^{h}$ which will be detailed in Section 2.5. Here, we try to focus on the formation of $\widehat{p}_{t+l^{h}}$ and $\widehat{\mathbf{V}}_{t+l^{h}}$ when $h=C$ and $h=F$.

With the belief of $h=C$, investor $h$ holds the idea that the historical price trend will continue; that is, $\widehat{p}_{t+l^{h}}=$ $\tilde{p}_{t-1} e^{\theta_{1} \widehat{r}_{t+l^{C}}^{h}}$, where $\widetilde{p}_{t-1}$ is settlement price of the last trading day; $\theta_{1} \sim U[0.1,1]$ is the intensity of chartist's belief responses to historical return $\widehat{r}_{t+l^{h}}^{C}=\ln \widetilde{p}_{t-1}-\ln \widetilde{p}_{t-l^{h}}$. In terms of $\widehat{\mathbf{V}}_{t+l^{h}}^{C}$, investor $h$ forms her expectation based on calculation over historical prices of stocks.

When $h=F$, investor $h$ believes that future prices will converge to future intrinsic values; that is, $\widehat{p}_{t+l^{h}}^{h}=p_{t+l^{h}, f}^{h}=$ $\tilde{p}_{t-1} e^{\theta_{2} \widehat{r}_{t+l^{h}}^{F}}$, in which $\widehat{r}_{t+l^{h}}^{F}$ is expected fundamental return based on information of future fundamental returns; $\theta_{1} \sim$ $U[0.1,1]$ is the reaction intensity of fundamentalist's belief. As for $\widehat{\mathbf{V}}_{t+l^{h}}^{F}$, its $i$ th row and $k$ th column element is given as $\widehat{\sigma}_{a}^{2}+\sum_{j=1}^{n} \widehat{\sigma}_{s_{j}}^{2}+\phi \widehat{\sigma}_{g_{i}}^{2}, \phi=1$ if $i=k$; otherwise $\phi=0$. After investor $h$ processed information and updated belief, she forms uncertainty perceptions about market and common and firm-specific factors, namely, $\widehat{\sigma}_{a}^{2}, \widehat{\sigma}_{s_{j}}^{2}$, and $\widehat{\sigma}_{g_{i}}^{2}$. Together with the formation of $\widehat{r}_{t+l^{h}}^{F}$, the belief updating process will be described in detail in Section 2.4.

2.4. Belief Updating with Constraint of Limited Capacity. In an ever-changing information environment of the stock market, investors have to constantly gather information and update their beliefs to adapt to the environment in order to construct a profitable portfolio. For $h=C$, the information needed to update belief is derived from historical prices, which are freely available from the market. For $h=F$, belief updating behavior needs analyzing information from all kinds of sources, like news, industry reports, and so on. This consumes some time and mental power, namely, information capacity in the present paper.

Sims $[8,9]$ first borrowed information entropy from the information theory [33] to describe limited information capacity of decision markers. Following these seminal papers, a large number of literatures [10-12] carry out in-depth studies concerning stock market with investors having limited information capacity. Take return of common factors in this paper, for example, the above-mentioned papers deem that uncertainty of posterior belief about $r_{s_{j}, t}$ must be smaller than that of prior belief after a signal about $r_{s_{j}, t}$ is received and that the amount of reduction in uncertainty equals consumed information capacity. According to [10], uncertainty of $r_{s_{j}, t}$ is the information entropy $H\left(r_{s_{j}, t}\right)=(1 / 2) \log _{2} 2 \pi e+\log _{2} \sigma_{s_{j}, t}$ scaled in bits with base 2 . The uncertainty of $\widehat{\mathbf{R}}^{F}$ is determined by its variance-covariance matrix; that is, $H\left(\widehat{\mathbf{R}}^{F}\right)=$ $(n / 2) \log _{2}(2 \pi e)+(1 / 2) \log _{2}\left(\operatorname{det}\left(\widehat{\mathbf{V}}^{F}\right)\right)[11,12]$, where $n$ is the number of stocks; $\operatorname{det}(\cdot)$ is the determinant operator.

In fact, the above literatures modeled limited information capacity of decision makers as the limited transiting power of information channels. At the same time, they assume that the extraction of signal must reduce uncertainty perception of investors about returns. This is modeling investors' behavior from perspective of learning, which does not apply to the belief updating case in this paper and is inconsistent with the fact that there is a lot of vague and even false information in the market. As the argument put forward by [7], understanding information is as much important as transiting or receiving information in communication. In the paper, limited information capacity of traders is measured as consumed information capacity in understanding the uncertainty. Since uncertainty is changing over time in a dynamic stock market, following [34] the present paper deems that the uncertainty perception of investors about fundamental returns may increase or decrease after acquiring and processing a piece of information. In either case, a certain amount of information capacity has to be consumed. We use absolute value of difference between uncertainty at trading days $t$ and $t+$ 1 ; that is, $\left|\log _{2}\left(\sigma_{t+1} / \sigma_{t}\right)\right|$ and $\left|(1 / 2) \log _{2}\left(\left(\operatorname{det} \mathbf{V}_{t+1}\right) /\left(\operatorname{det} \mathbf{V}_{t}\right)\right)\right|$ to quantify consumed capacity of investors during their adaption from trading day $t$ to $t+1$.

In the following, we will explain the process of information processing and belief updating when $h=F$. For simplicity, notations with the same subscript, that is, $\sigma_{i}^{2}(i=$ $1,2, \ldots, 1+m+n)$, successively denote variances of the market factor, $m$ firm-specific factors, and $n$ common factors. Since there are two distinctive phases including belief updating about risk factors and portfolio construction based on returns, we divide information processing and capacity consumption into $\left|\log _{2}\left(\sigma_{i, t+1} / \sigma_{i, t}\right)\right|$ and $\left|(1 / 2) \log _{2}\left(\left(\operatorname{det} \mathbf{V}_{t+1}\right) /\left(\operatorname{det} \mathbf{V}_{t}\right)\right)\right|$, to which investor $h$ allocates the fraction $\eta_{1}$ and $\eta_{2}$ of her total capacity $\kappa^{h}>0$. That is, $\kappa^{h}=\eta_{1} \kappa^{h}+\eta_{2} \kappa^{h}=\kappa_{q}^{h}+\kappa_{p}^{h}$ and $\eta_{1}+\eta_{2}=1$.

Once investor $h$ enters the market at trading day $t$, she gets access to information about future fundamental returns of $1+$ $m+n$ factors over the interval $\left[t, t+l^{h}\right]$ from various sources, such as industry reports and news, at probability $\beta \in[0,1]$ 
indicating market transparency. First, investor $h$ processes information concerning returns at trading day $t+1$ for all risk factors; then she moves on to trading day $t+2$, and so on. However, whether investor $h$ could process all information until $t+l^{h}$ depends on her information capacity. Therefore, the actual time window length $\bar{l}^{h}$ over which investor $h$ can process all information must satisfy $\widetilde{l}^{h} \leq l^{h}$.

From (2), we know that $\mathbf{V}_{t}^{h}$ is composed of $\sigma_{i}^{2}$; thus $\mathbf{V}_{t}^{h}$ evolves to $\widehat{\mathbf{V}}_{t}^{h}$ after investor $h$ updates her uncertainty perception about risk factors. Therefore, the updating behavior of investor $h$ is constrained not only by the information capacity $\kappa_{q}^{h}$ concerning risk factors, but also by $\kappa_{p}^{h}$ with regard to return vector. In other words, two inequalities given in expression (6) must not be violated during belief updating.

$$
\begin{gathered}
\frac{1}{2} \sum_{j=1}^{\tilde{l}^{h}} \sum_{i=1}^{1+m+n}\left|\log _{2}\left(\frac{\sigma_{i, t+j}}{\sigma_{i, t+j-1}}\right)\right| \leq k_{q}^{h}, \\
\frac{1}{2} \sum_{j=1}^{\eta^{h}} \sum_{i=1}^{1+m+n}\left|\log _{2}\left(\frac{\left(\operatorname{det} \widehat{\mathbf{V}}_{t}^{h}\right)}{\left(\operatorname{det} \mathbf{V}_{t}^{h}\right)}\right)\right| \leq k_{p}^{h} .
\end{gathered}
$$

In simulation, after belief updating investor $h$ applies the realizations $\tilde{r}_{s_{j}}$ generated from $r_{s_{j}} \sim N\left(\mu_{s_{j}}, \sigma_{s_{j}}^{2}\right)$ and according variances to expectation formation, namely, $\widehat{r}_{s_{j}, t+l^{h}}^{F}=$ $\left(1 / \widetilde{l}^{F}\right) \sum_{l^{h}=1}^{\widetilde{l}^{F}} \widetilde{r}_{s_{j}, t+l^{h}}$ and $\widehat{\sigma}_{s_{j}, t}^{2}=\left(1 / \widetilde{l}^{F}\right) \sum_{l^{h}=1}^{\widetilde{l}^{F}} \sigma_{s_{j}, t+l^{h}}^{2}$, it can be seen that the magnitude and uncertainty of $\tilde{r}_{s_{j}}$ are positively correlated with $\mu_{s_{j}}$ and $\sigma_{s_{j}}^{2}$, which are referred to as information content and information amount in the present paper, respectively.

2.5. Order Formation and Clearing Mechanism. Continuous double auction (CDA) is introduced as clearing and pricing mechanism. The operation of this mechanism requires investors to submit orders with elements of timing, quote, quantity, and trade direction. After investors enter the market sequentially and randomly, they form the quote and optimal portfolio under the constraint of resources, that is, no short selling and no short buying. Then order size and trade direction are determined given current shares of holding stocks. For the sake of brevity, we discard the subscript in the analysis that applies to every stock.

The formation of quote is affected by price range revealed by order book, expected price, and resources. After entering the market, investor $h$ observes the price range $\left[p_{\tau}-10 \Delta, p_{\tau}+\right.$ $10 \Delta$ ] revealed by the book, where $p_{\tau}$ is the last transaction price at intraday time $\tau \in R^{+}$or $p_{\tau}=\widetilde{p}_{t-1}$ if no transaction happens; $\Delta=0.01$ is the tick size. After randomly drawing a price $p_{b, \tau}^{h} \sim U\left[p_{\tau}-10 \Delta, p_{\tau}+10 \Delta\right]$, the quote is preliminary determined as $p=p_{b, \tau}^{h}+0.1 \times\left(\widehat{p}_{t+l^{h}}-p_{\tau}\right)$, from which we can see that $p$ is higher if $\widehat{p}_{t+l^{h}}$ is greater than $p_{\tau}$, and vice versa. This is consistent with behavior of investors in real-life stock market. When it comes to only one stock, according to [32], we can obtain the only highest quote $p_{o, \max }^{h}=\widehat{p}_{t+l}^{h}$ and the only lowest quote $0<p_{o \text {, min }}^{h}<p_{o}^{h}$ by taking resources constraint into account. Thus, the quote $p_{o}^{h}$ is finally given in

$$
p_{o}^{h}= \begin{cases}p_{o, \max }^{h} & \text { if } p>p_{o, \max }^{h} \\ p_{o, \min }^{h} & \text { if } p<p_{o, \min }^{h} \\ p & \text { else. }\end{cases}
$$

Let $w_{i, t}^{h}(i=1, \ldots, m)$ be the fraction of wealth expected to be invested into each stock. At this point, $w_{i, t}^{h}(i=1, \ldots, m)$ can be computed separately according to (5). However, considering resources constraint faced by entire portfolio, investor $h$ only distributes a fraction of total wealth $W_{t}^{h}$ to $i$ th stock with $w_{i, t}^{h}>0$. Thus, actual wealth invested into $i$ th stock is $z_{i, t}^{h}=W_{t}^{h} w_{i, t}^{h} / \sum w_{i, t}^{h}\left(w_{i, t}^{h}>0\right)$. Thus, the shares of $i$ th stock that investor $h$ would like to hold are $d_{i, t}^{h}=\left[z_{i, t}^{h} / p_{i, o}^{h}\right]$, where $[\cdot]$ is the operator of rounding down to the nearest integer. By comparing the demanding shares $d_{i, t}^{h}$ and the shares that investor $h$ already holds $q_{i, t}^{h}$, order size and trade direction are given in (8), where $1,-1$, and 0 represent buying, selling, and holding current position.

$$
\begin{aligned}
& v_{i, t}^{h}=1, \\
& o_{i, t}^{h}=d_{i, t}^{h}-q_{i, t}^{h} \\
& \text { if } d_{i, t}^{h}>q_{i, t}^{h} \\
& v_{i, t}^{h}=-1, \\
& o_{i, t}^{h}=q_{i, t}^{h}-d_{i, t}^{h} \\
& v_{i, t}^{h}=0, \quad \text { if } d_{i, t}^{h}<q_{i, t}^{h} \\
& o_{i, t}^{h}=0 \quad \text { if } d_{i, t}^{h}=q_{i, t}^{h} .
\end{aligned}
$$

Orders are stored in the book with priority of price first and then with the priority of timing if the prices of orders are the same. The condition of transaction is met if the highest quote of orders on the buying side is greater than the lowest quote of orders on the selling side. The matched buying (selling) order is the one with highest (lowest) quote and with earlier submission time if quotes are the same for more than one order on the same side.

The traded volume of the transaction equals the smaller size of matched orders, and the transaction price is the quote of earlier submitted one of matched orders. After transaction, corresponding shares of stock and cash are added to or subtracted from positions of investors. At the same time, the matched order with smaller size is cleared from the book, while the remaining fraction of the bigger order is retained in the book. See $[34,35]$ for more technical details of the CDA mechanism. Settlement price of $i$ th stock at trading day $t$, that is, $\widetilde{p}_{i, t}$, is the last transaction price at that day. 
TABLE 1: Parameters and descriptions.

The number of stocks

Initial intrinsic values and prices of stocks

Changing intensity of the market and firm-specific factors

Information efficiency of the market

The population of investors

Initial holding shares of stocks

Information capacity of investors

Weight of information capacity allocation

Information content of the market factor

Information content of firm-specific factors

Information amount of the market factor

Information amount of firm-specific factors

$$
\begin{gathered}
m=3 \\
\widetilde{p}_{i, 0}=p_{i, 0, f}=10 \\
\alpha_{a}=1 / 60, \alpha_{g}=1 / 20 \\
\beta=0.5 \\
N=5000 \\
q_{i, 0} \sim U[20,50] \\
K^{h} \sim U[1,1.5] \\
\eta_{1}=0.8, \eta_{2}=0.2 \\
{\left[\mu_{a, \min }, \mu_{a, \max }\right]=[-3,3] \times 10^{-5}} \\
{\left[\mu_{g, \min }, \mu_{g, \max }\right]=[-1.2,1.2] \times 10^{-4}} \\
{\left[\sigma_{a, \min }^{2}, \sigma_{a, \max }^{2}\right]=[2,4] \times 10^{-6}} \\
{\left[\sigma_{g, \min }^{2}, \sigma_{g, \max }^{2}\right]=[2,2.2] \times 10^{-5}}
\end{gathered}
$$

\section{Simulation Results and Discussion}

3.1. A Typical Simulation Result and Model Validation. Before simulation, we first assign values and ranges to parameters that will not change in the following. Those parameters in Table 1 denote traits of investors and economic environment of the artificial stock market.

In the present paper, we focus on effects of information characteristics relevant to common factors on the dynamics of stocks traded by investors with limited information capacity. In order to perform a typical simulation to show basic results of the model, parameters representing those characteristics are assigned with benchmark values that will vary in following in-depth studies. Those parameters are the number of information sources about common factors $n=4$, dynamic intensity of information $\alpha_{s}=1 / 40$, information content $\left[\mu_{s, \min }, \mu_{s, \max }\right]=[-8,8] \times 10^{-5}$, and information amount $\left[\sigma_{s, \text { min }}^{2}, \sigma_{s, \text { max }}^{2}\right]=[1.2,1.4] \times 10^{-5}$. Information content and amount of common factors are set at quite small values to keep stocks' intrinsic values within a reasonable magnitude. This will not affect our main results.

Based on parameters shown in Table 1, we conduct a typical simulation. Then, reproduced time series are presented and their features are analyzed. On the one hand, we can see how intrinsic values of stocks affect dynamics of prices. On the other hand, we can validate the model by examining whether reproduced time series have stylized facts of prices and returns in the real-world stock market.

Figure 1 presents dynamics of three simulated stock prices and exogenously given intrinsic values composed of realizations of former mentioned risk factors. A total number of 1600 time steps are divided into several stages for illustration purpose. Because the maximum of investors' time window length is 200, the first 200 history intrinsic values are taken as substitutes for history prices of stocks providing an extrapolating basis for chartist's belief during run-in steps (time steps from 201 to 400 ). The last 200 intrinsic values provide information about future fundamental returns to fundamentalist's belief functioning from time step 1201 to 1400. As run-in steps, simulated prices from time step 201 to 400 are discarded in our analysis, because those simulated

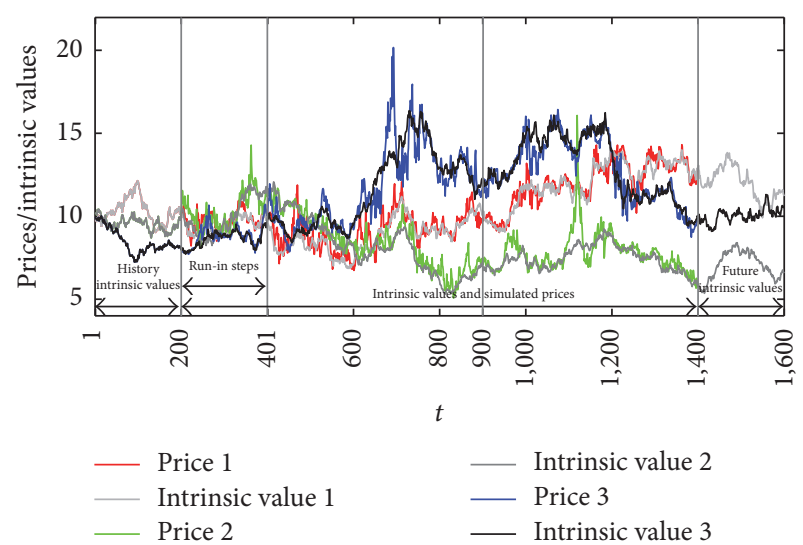

FIGURE 1: Intrinsic values and simulated prices of stocks.

prices are formed based on exogenously given intrinsic values rather than endogenous simulated history prices.

Thus, our analysis in this and following sections are aimed at simulated results of 1000 time steps from 401 to 1400 . From Figure 1, we can see that prices track closely around intrinsic values, and their volatilities show higher degree of fluctuation beyond those of intrinsic values. The fluctuations of prices are more prominent when intrinsic values rise and fall significantly.

Selected returns of intrinsic values and simulated prices are displayed in Figures 2(a) and 2(b) separately. By comparing two figures, we observe that returns of prices are featured with volatility clustering; that is, large fluctuation is followed by large ones and small fluctuation is followed by small ones. Q-Q plots in Figure 3 also demonstrate that returns of all three stocks have peakedness and fat-tailed distributions with kurtosis of 4.8643, 7.1420, and 7.6535. Meanwhile, as shown in autocorrelation diagrams inserted in Figure 3, the absolute and squared returns of prices have the behavior of long memory. Aside from stylized facts in returns, the main trends of prices are determined by dynamics of intrinsic values, although there are some temporary deviations of prices from intrinsic values caused by the chartist's belief. Based on these 

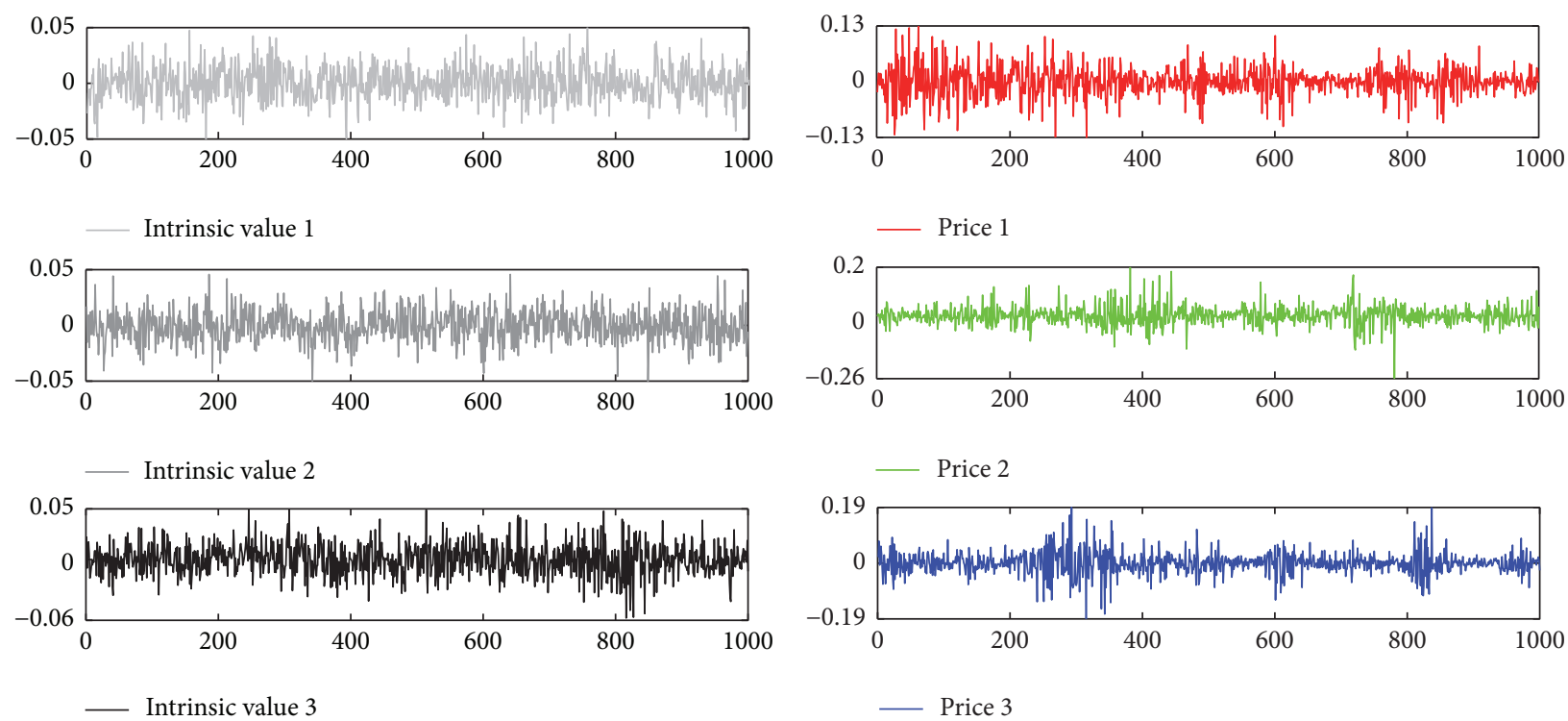

(a)

(b)

FIgURE 2: Time series of returns of intrinsic values and prices.

results, we can conclude that, with given information characteristics, microstructure, and limited information capacity of investors, the present multiagent-based model can reproduce prices and returns whose dynamics consisted of our common knowledge and having stylized facts observed in real-world stock market.

3.2. Uncertainty. Uncertainty is one of the most important indicators for dynamics of stock returns. Variance is a commonly used measurement for uncertainty, but there are some limitations such as its sensitivity to magnitude of sample data. Taking the case of two binary variables $X$ and $Y$ as an example, $X$ is realized as 1 and -1 with equal probability, while $Y$ is realized as 0.1 and -0.1 also with equal probability. Obviously, the variance of data consisting of random realizations of $X$ is much greater than that of $Y$. However, this does not mean the uncertainty of $X$ is greater than $Y$, if we are endowed with the ex ante belief of binary distributions. In fact, Shannon [36] first introduces information entropy, which is only determined by the probability distribution of concerned variable, into the field of the information theory to measure this uncertainty.

According to the results shown in Figure 1, we can see that the prices track closely around intrinsic values and that the fluctuation of prices is large when the intrinsic values are more fluctuant. Then, it is reasonable to assume that the greater the volatility of intrinsic values caused by common factors, the greater the volatility that simulated stock prices exhibit. However, this assumption holds only when uncertainty contained in information about common factors is received and understood by investors and then transformed to information-bearing orders.

In the previous subsection, we validate the effectiveness of information-based fundamentalist's belief and microstructure of the market in impounding of fundamental returns into prices under basic model parameter settings. In this subsection, we proceed to investigate the adaptive behavior of traders to dynamic characteristics of common factors and its effects on stock dynamics. Those characteristics include the number of information sources of common factors $n$, increasing dynamic intensity $\alpha_{s}=1 /\left(60-4 n_{\alpha}\right)\left(n_{\alpha}=1,2, \ldots, 10\right)$, more indicative information content $\left[\mu_{s, \min }, \mu_{s, \max }\right]=[-2-$ $\left.n_{\mu}, 2+n_{\mu}\right] \times 10^{-5} \quad\left(n_{\mu}=1,2, \ldots, 10\right)$, and larger information amount $\left[\sigma_{s, \text { min }}^{2}, \sigma_{s, \max }^{2}\right]=\left[2,2+2 n_{\sigma}\right] \times 10^{-6}\left(n_{\sigma}=1,2, \ldots, 10\right)$.

Information entropy is employed to measure uncertainty of returns of intrinsic values and simulated prices. Obviously, realizations of intrinsic values and simulated prices are discrete. For discrete variable $X$, its information entropy is $H(X)=-\sum_{x} p(x) \log _{2} p(x)$, which is determined only by the probability distribution $p(x)$. To calculate information entropy of returns, we have to classify all data points of time series into several nonoverlapping states; and frequencies of returns falling into each state are viewed as probability distribution in calculation. According to magnitudes of returns displayed in Figure 2, we shall apply different criteria in dividing the ranges of returns of intrinsic values and prices. In order to make a comparative analysis, the number of states should be the same, because with the same number of states $n_{s}$ the information entropy of any time series has the same upper limit $-\sum_{i=1}^{n_{s}}\left(1 / n_{s}\right) \log _{2}\left(1 / n_{s}\right)$. We use eight real numbers to divide ranges of returns of intrinsic values and prices into nine states. Smaller and greater numerical divisions for intrinsic values and prices are employed, respectively; that is $\lambda_{f}=\{-0.07,-0.05,-0.03,-0.01,0.01,0.03,0.05,0.07\}$ for intrinsic for intrinsic values and $\lambda_{c}=3 \lambda_{f}$ for simulated prices.

Under the environment of these changing information characteristics, simulations are performed with $n=2$ and $n=4$ separately. Figure 4 shows changing trends of total 


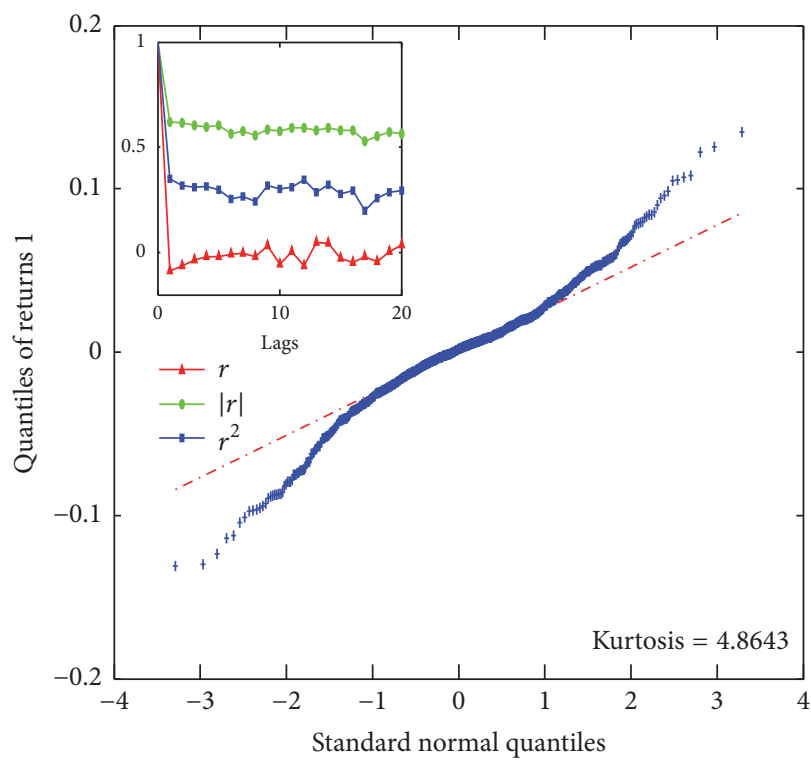

(a)

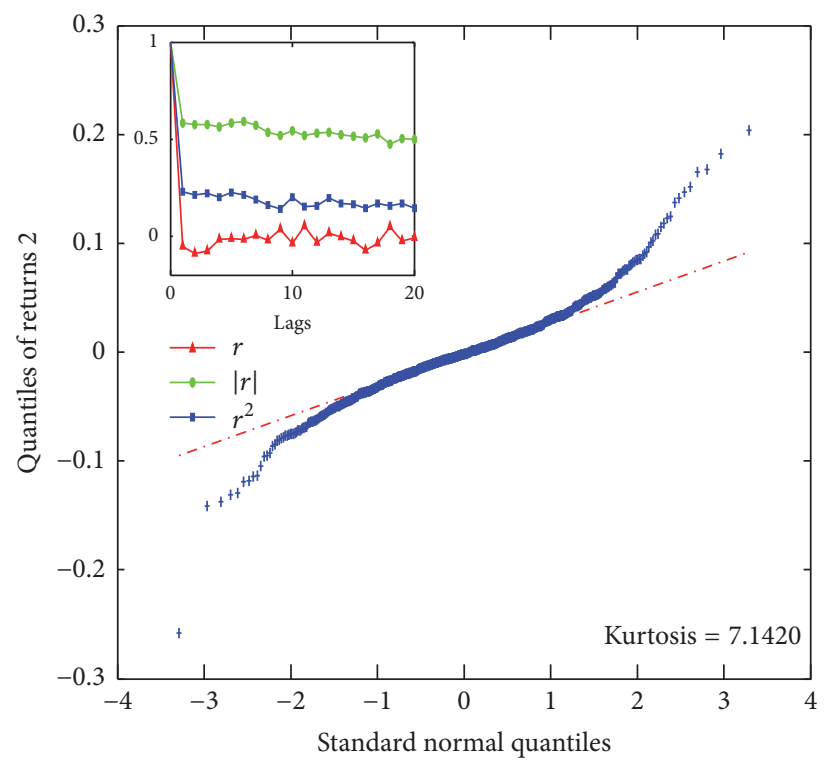

(b)

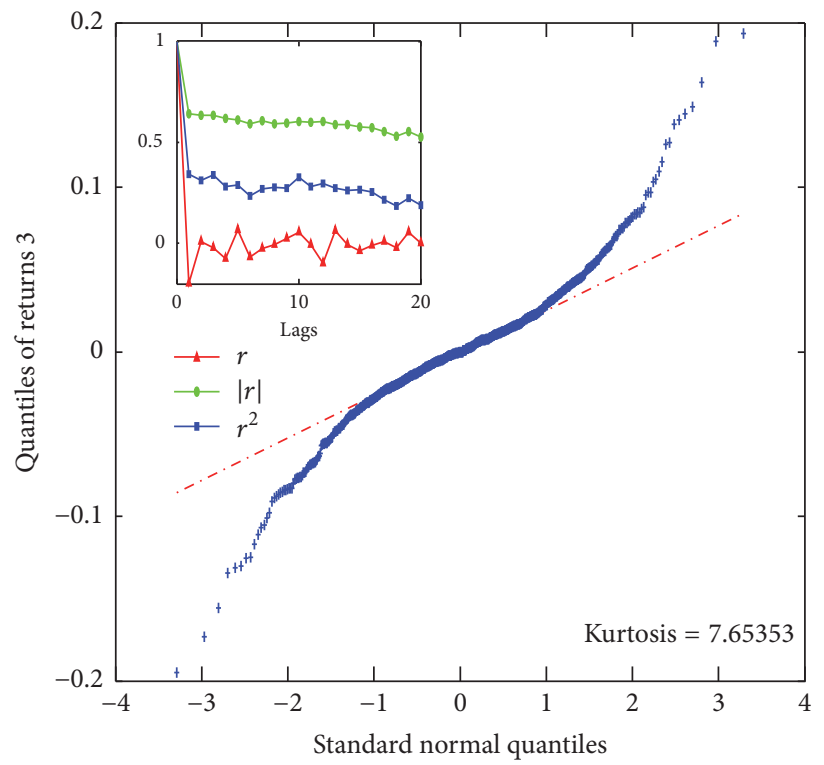

(c)

FIGURE 3: Q-Q plots about returns of stock prices and autocorrelation diagrams of raw, absolute, and squared returns.

information entropy of returns of intrinsic values $\mathrm{TH}\left(r^{f}\right)=$ $\sum_{i=1}^{3} H\left(r_{i}^{f}\right)$, those of prices $\mathrm{TH}\left(r^{p}\right)=\sum_{i=1}^{3} H\left(r_{i}^{p}\right)$, and relative uncertainty of returns of prices to intrinsic values $R_{\mathrm{TH}}=$ $\mathrm{TH}\left(r^{p}\right) / \mathrm{TH}\left(r^{f}\right)$. In order to ensure the robustness, results here and below are average values of results generated from 20 times simulations with different random seeds. We can see from Figure 4 that $R_{\mathrm{TH}}$ exhibits different features because of different dynamics of price returns and similar dynamic shared by intrinsic values. Increases of these three kinds of information characteristics all contribute to the increase of uncertainty of returns of prices. The result suggests that same level of market uncertainty may result from very different characteristics of common factors. At the same time, in all three information environments the uncertainty when $n=4$ is greater than the uncertainty when $n=2$.

As intensity increases, it is harder for investors to adapt to more dynamic information environment and to understand information about fundamental returns accurately. It means that small amount of information disclosed at high intensity may consume large information capacity and cause increased price uncertainty as shown in Figure 4(a).

The greater absolute value of the information content does not consume more information capacity of investors. However, it has much more explicit implication and prompts traders to form aggressive expected prices, as shown in Figure 4(b). As illustrated in inserted plot in Figure 4(c), 


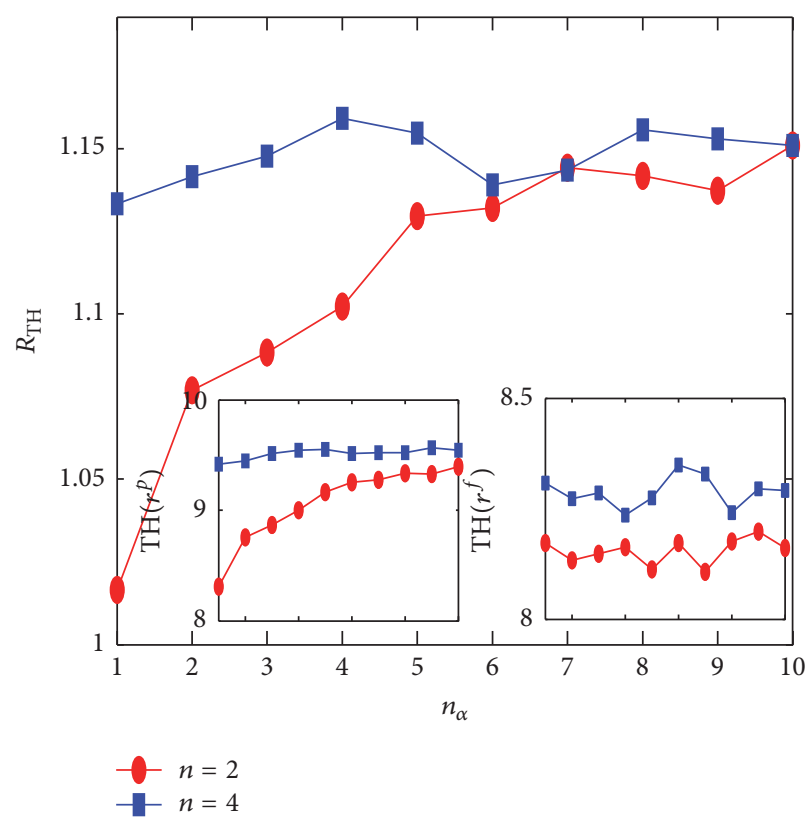

(a)

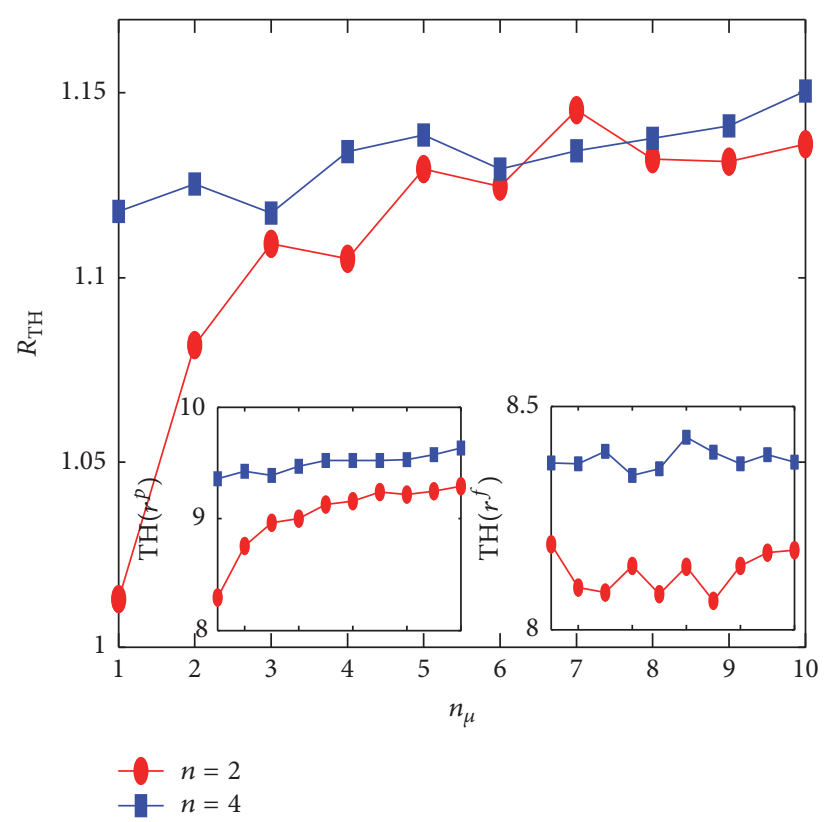

(b)

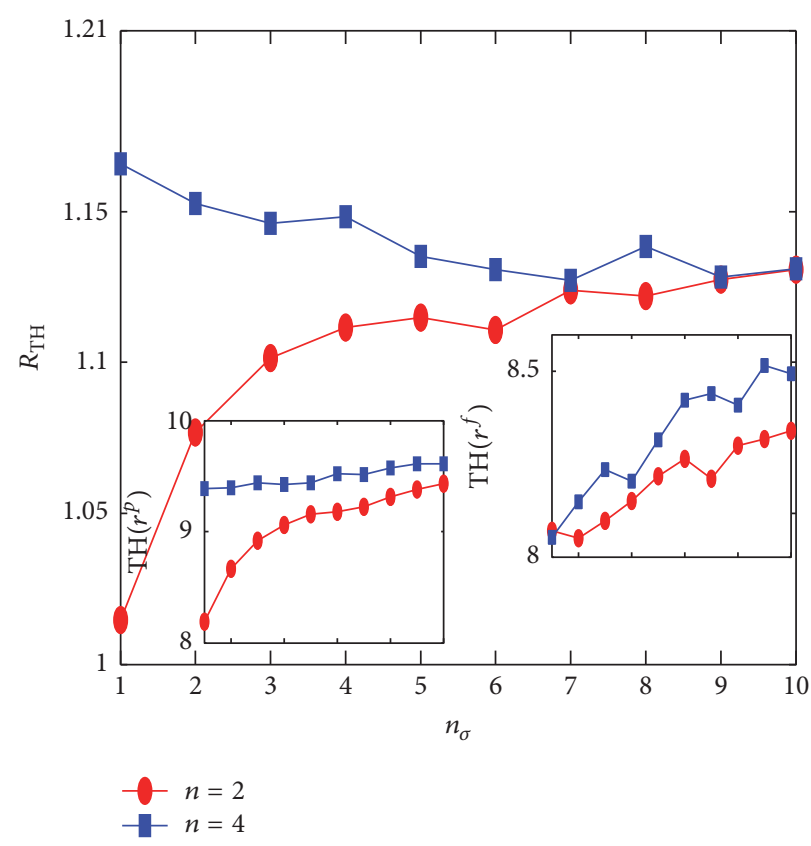

(c)

FIGURE 4: Uncertainty and relative uncertainty against changes of different information characteristics of common factors.

different from dynamic intensity and information content the uncertainty of returns of intrinsic values increases with the increase of information amount. However, the increasing uncertainty trends of returns of prices do not differ much from trends generated from other two information characteristics. This is because larger amount of uncertainty contained in intrinsic values overloads information capacity of investors. They cannot understand the uncertainty and thus cannot update their beliefs accordingly. In consequence, the uncertainty is not incorporated into trading behavior, which is the only way to cause price fluctuations. The same reason also explains why more information sources of common factors do not lead to proportional increases in uncertainty.

The above results imply that limited information capacity of traders may cause market inefficiency, and it is more severe in the presence of more information sources. It also suggests that the explicitness of information and means of 
information disclosure also affect stock dynamics and market efficiency.

3.3. Dependence. As a special type of risk factors, common factors not only affect uncertainty of returns but more importantly also affect dependence between them. In this subsection, we turn to study changes of dependence caused by the same changes in information characteristics in the previous subsection. As for measurement for relation between stocks, Pearson linear correlation coefficient is the most common indicator. However, this is not accurate, faced with widespread nonlinear behavior in stock market [37-40]. It has been demonstrated that mutual information, which also borrowed from the information theory, can capture nonlinear dependence between time series [40-43].

The mutual information of $X$ and $Y$ is defined as $\operatorname{MI}(X, Y)=H(X)+H(Y)-H(X, Y)$, where $H(X, Y)=$ $-\sum_{x} \sum_{y} p(x, y) \log _{2} p(x, y)$ is joint entropy of $X$ and $Y$ determined by joint probability distribution $p(x, y)$. It can be seen that the greater $\operatorname{MI}(X, Y)$ is, the more the information about $Y(X)$ is contained in $X(Y)$ and the stronger the dependence between $X$ and $Y$ is. In order to calculate the MI between two random variables, we have to compute joint probability distribution of variables first. It has been proven that the division that keeps frequencies of returns falling into all states the same is an effective method [34]. Therefore, we use eight quantiles to divide the range of returns into nine states, and the set of quantiles is $q=\left\{q_{1 / 9}, q_{2 / 9}\right.$, $\left.q_{3 / 9}, q_{4 / 9}, q_{5 / 9}, q_{6 / 9}, q_{7 / 9}, q_{8 / 9}\right\}$ indicating quantiles $1 / 9,2 / 9$, and so on.

Against changing of different information characteristics of common factors, Figure 5 shows the overall dependence between returns of simulated prices and intrinsic values, that is, $\operatorname{TMI}\left(r^{p}\right)=\sum_{i, j=1}^{3} \operatorname{MI}\left(r_{i}^{p}, r_{j}^{p}\right)(i>j)$ and $\operatorname{TMI}\left(r^{f}\right)=$ $\sum_{i, j=1}^{3} \operatorname{MI}\left(r_{i}^{f}, r_{j}^{f}\right)(i>j)$, and relative dependence of price returns to intrinsic value returns, that is, $R_{\mathrm{TMI}}=\mathrm{TMI}\left(r^{p}\right) /$ $\operatorname{TMI}\left(r^{f}\right)$. The results indicate that similar dependence between stocks may be caused by different intrinsic relation in the market. Trends of dependence concerning intrinsic values are similar to those of uncertainty. However, there exists a discrepancy between trends of dependence and trends of uncertainty. Unlike instability in growth that uncertainty of prices showed, dependence between returns of prices increases at a stable speed. This is because the behavior of portfolio optimization in the present paper more easily causes dependence between returns. As implied in (5), belief updating based on information of any risk factor, not just common factors, will affect demands for all stocks via variance-covariance matrix.

3.4. Relation between Uncertainty and Dependence. From the above results, we know that changes of information characteristics of common factors lead to increases in uncertainty of returns of prices as well as dependence between them. In other words, extraction of common factors will improve analysis efficiency in portfolio construction, but its application increases dependence between stocks in that portfolio at the same time, which could impair the benefit of diversification brought by portfolio optimization. In this subsection, we focus on the analysis of relation between uncertainty and dependence against changes of different information characteristics of common factors. We consider two forms of relation between uncertainty and dependence: relation between uncertainty $\operatorname{TH}\left(r^{p}\right)$ and dependence $\operatorname{TMI}\left(r^{p}\right)$ and relation between relative uncertainty $R_{\mathrm{TH}}=\mathrm{TH}\left(r^{p}\right) / \mathrm{TH}\left(r^{f}\right)$ and relative dependence $R_{\mathrm{TMI}}=\mathrm{TMI}\left(r^{p}\right) / \mathrm{TMI}\left(r^{f}\right)$.

Figure 6 shows relation between uncertainty and dependence with changes of different characteristics of common factors. Scatter plots illustrate strong positive relation between them, which consisted of findings in [44]. Particularly when $n=4$, scattered points are fitted by straight lines with high goodness of fit. In addition, we can see from embedded plots that dependence grows faster than uncertainty against increases of information characteristics of common factors. The result indicates that changes of information characteristics lead to more dependence than uncertainty in returns of prices and imply that the application of common factor analysis provides explanatory power but bring consequence of excessive comovement at the same time.

In previous subsections, we found that trends of uncertainty and dependence pertinent to simulated prices have similar increasing tendency, while trends of uncertainty and dependence concerning returns of intrinsic values exhibit different features. Therefore, in order to get an in-depth understanding of effects of information characteristics of common factors on dynamics of prices, we shall take into account in our analysis the underlying returns of intrinsic values, based on which simulated prices are generated.

As shown in Figure 7, the relative uncertainty $R_{\mathrm{TH}}=$ $\mathrm{TH}\left(r^{p}\right) / \mathrm{TH}\left(r^{f}\right)$ and relative dependence $R_{\mathrm{TMI}}=\mathrm{TMI}\left(r^{p}\right) /$ $\operatorname{TMI}\left(r^{f}\right)$ are still positively correlated. However, these positive correlations are greatly weakened as indicated by both scatter plots and goodness of linear fitting. Meanwhile, we can see from inserted plots that, compared with the case of $n=2$, the increase of relative dependence is slower than the increase of relative uncertainty when $n=4$. This is especially prominent with increases of information amount of common factors as shown in Figure 7(c). The result implies that the technique of extraction and application of common factors is more effective in the information environment with a large number of information sources and large information amount.

\section{Robustness}

In this section, we would like to provide robustness check of the model. The main purpose of the present paper is investigating effects of common factors on dynamics of stocks traded by investors with limited capacity. Since changing characteristics of common factors are studied in previous sections, here we focus on information capacity of traders. The concerned variables are information capacity $K^{h}$ and allocation weights $\eta_{1}$ and $\eta_{2}$, which are set as $K^{h} \sim U[1,1.5]$ and $\eta_{1}=0.8, \eta_{2}=0.2$ previously. To check the robustness, 

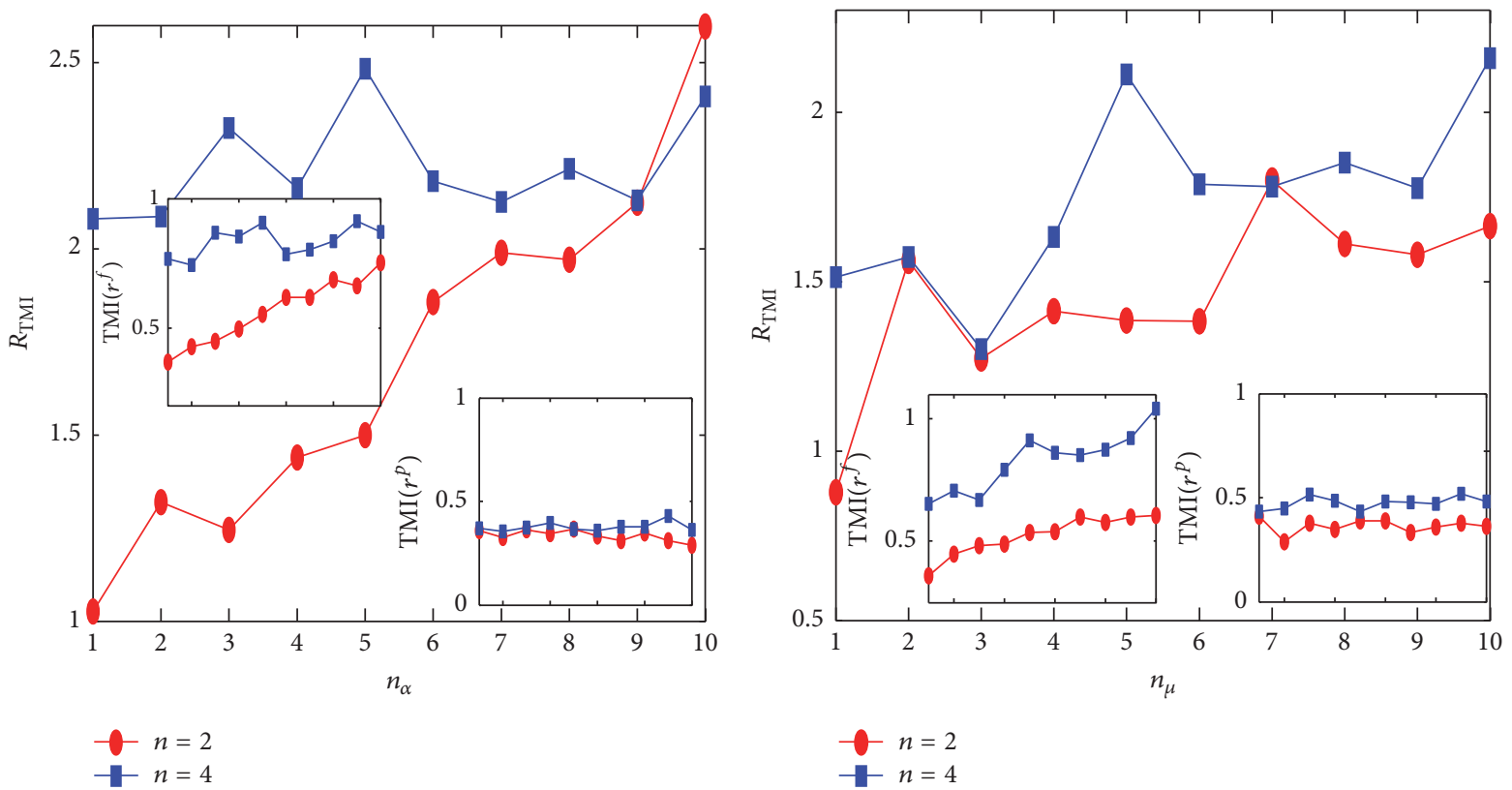

(a)

(b)

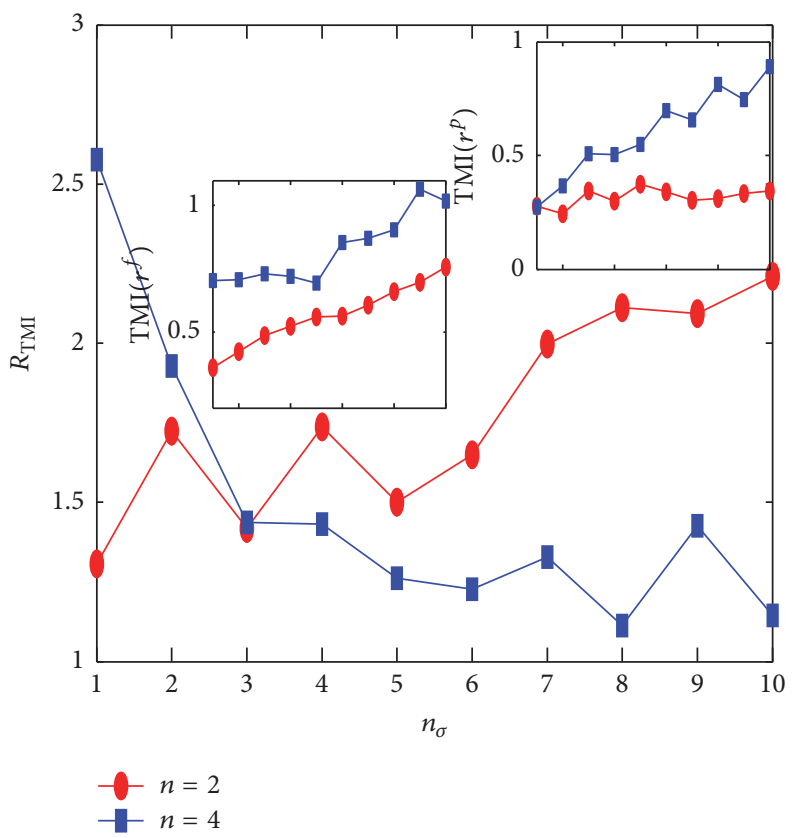

(c)

FIGURE 5: Dependence and relative dependence against changes of different information characteristics of common factors.

we follow [35] and adjust the parameters to observe the simulation results of other cases, as different combinations of parameter values shown in Table 2 .

Compared to Case 5 already studied in previous sections, other cases can be considered as the ones with higher or lower information capacity and more symmetric or asymmetric allocation weights. In order to make comparative analysis, only simulation results of Case 2, Case 4, Case 6, and Case 8 are reported. In consideration of brevity, we just report in Figure 8 simulation results concerning changing intensities of common factors as displayed in Figures 4(a) and 6(a). From
Figure 8 we know that the main results are the same as those of the benchmark case. Simulation results with changes in other information characteristics of common factors, which are not reported here, also consisted of the benchmark case. Thus, our model is robust with moderate changes of parameter values.

\section{Conclusion}

We developed an artificial stock market in which heterogeneous investors update their beliefs when faced with the 

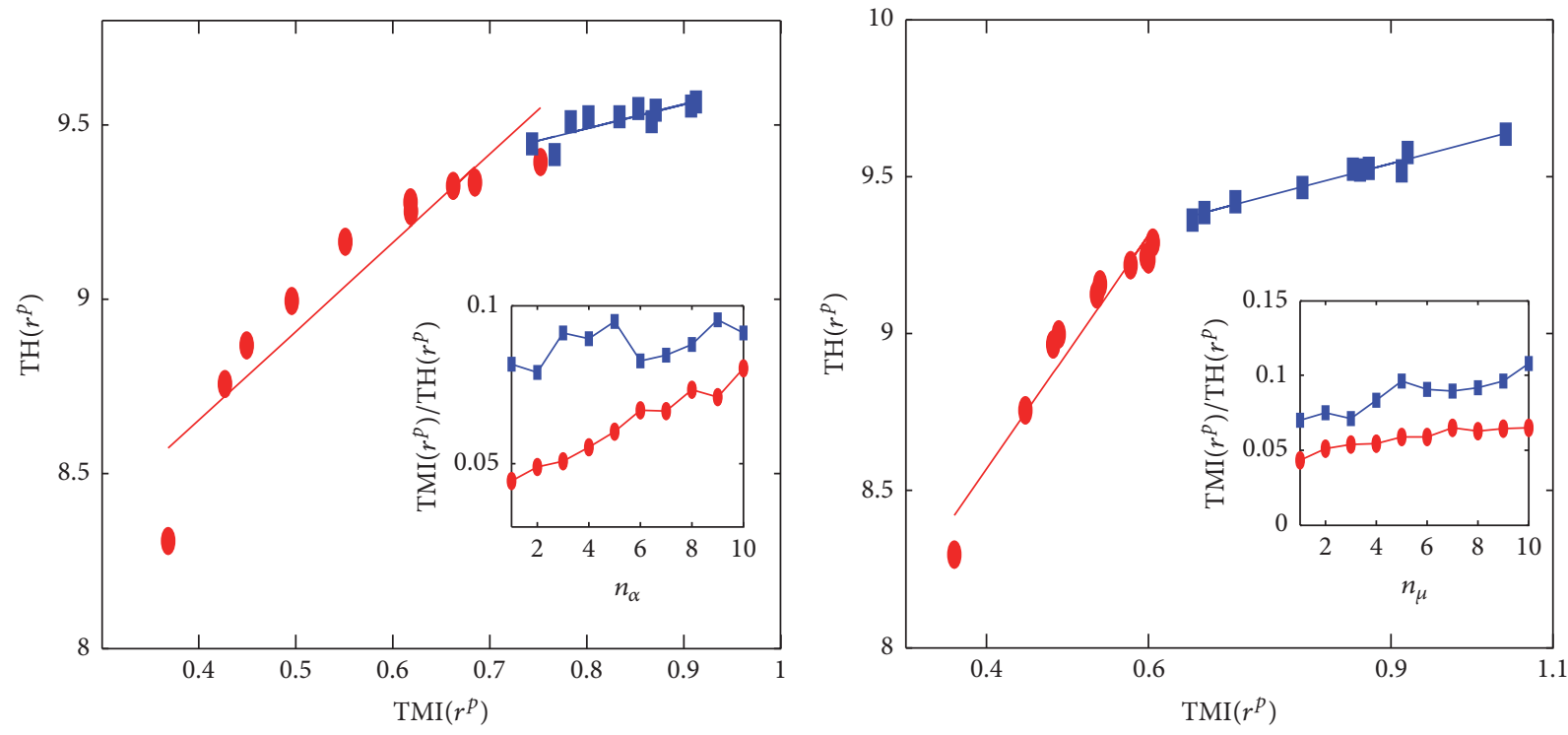
$n=2$
$n=2$
$n=4$
$n=4$

(a)

(b)

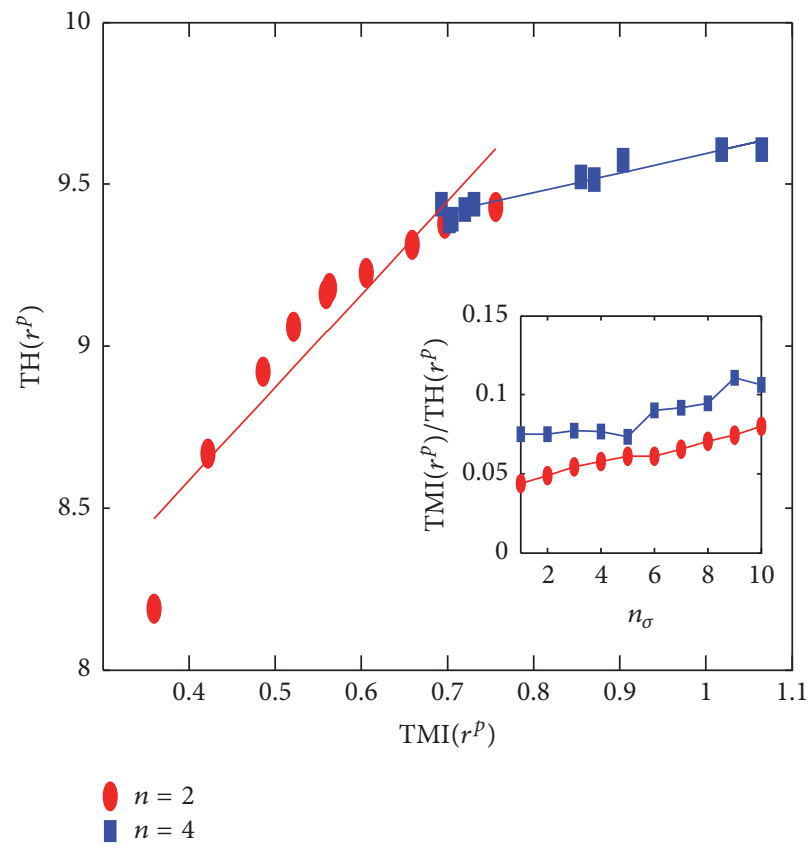

(c)

FIgURE 6: Relation between uncertainty and dependence.

TABLE 2: Different parameter combinations of information capacity and allocation weights.

\begin{tabular}{lccccccccc}
\hline Cases & Case 1 & Case 2 & Case 3 & Case 4 & Case 5 & Case 6 & Case 7 & Case 8 & Case 9 \\
\hline Allocation weights & $\eta_{1}=0.9$ & $\eta_{1}=0.8$ & $\eta_{1}=0.7$ & $\eta_{1}=0.9$ & $\eta_{1}=0.8$ & $\eta_{1}=0.7$ & $\eta_{1}=0.9$ & $\eta_{1}=0.8$ & $\eta_{1}=0.7$ \\
& $\eta_{2}=0.1$ & $\eta_{2}=0.2$ & $\eta_{2}=0.3$ & $\eta_{2}=0.1$ & $\eta_{2}=0.2$ & $\eta_{2}=0.3$ & $\eta_{2}=0.1$ & $\eta_{2}=0.2$ & $\eta_{2}=0.3$ \\
Information capacity & & $K^{h} \sim U[0.5,1]$ & & & $K^{h} \sim U[1,1.5]$ & & & $K^{h} \sim U[1.5,2]$ & \\
\hline
\end{tabular}




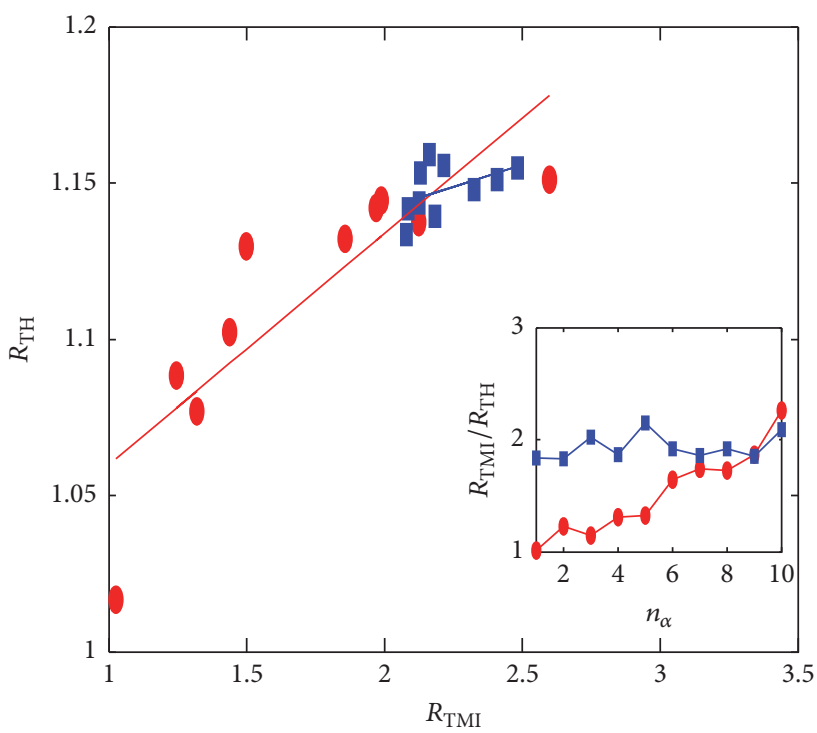

$n=2$

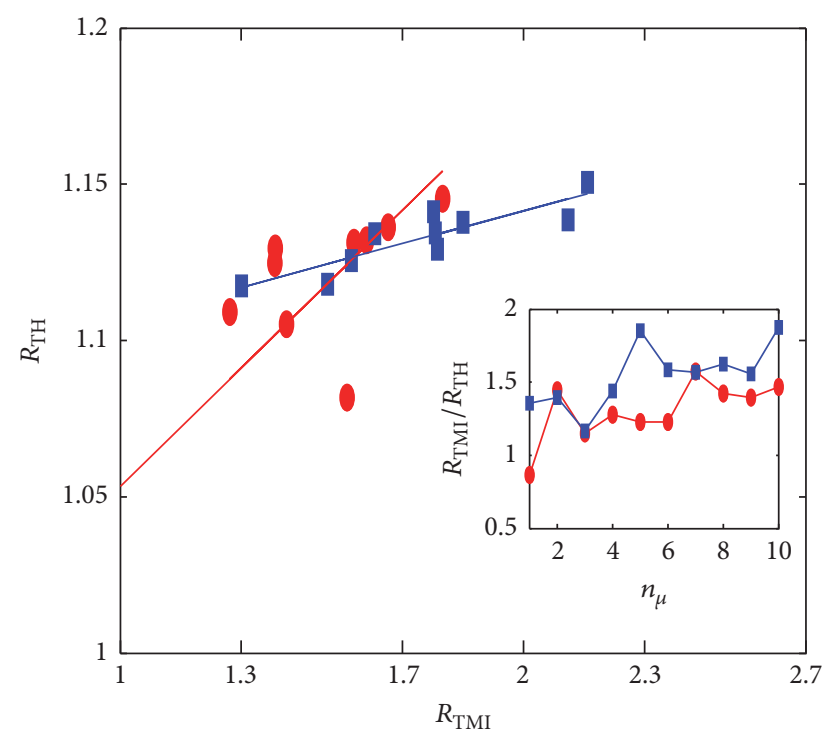

$n=2$

(a)

(b)

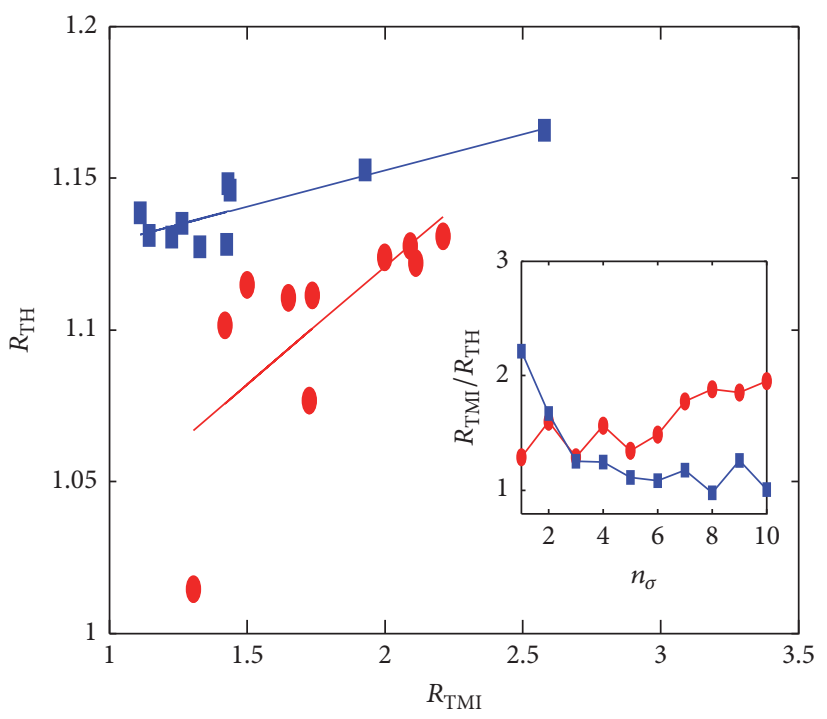

$n=2$

$n=4$

(c)

FIGURE 7: Relation between relative uncertainty and relative dependence.

constraint of limited information capacity. By updating beliefs, investors adapt to the dynamic changes in characteristics of common risk factors and optimize their portfolios accordingly. The results show that the agent-based model in the present paper can reproduce stock prices with realistic features; namely, the general evolution of prices is determined by the intrinsic values, and the returns of simulated prices are featured with some stylized facts such as volatility clustering, peakedness, and fat-tailed distribution. By varying parameters in the basic model, we proceed to study effects of changing information characteristics of common factors which have on dynamics of stocks. It is found that uncertainty and dependency related to prices both increase along with changing information environment, but the increasing trends are constrained by the limited information capacity of investors. Increasing trends of uncertainty and dependency are positively correlated, and the increase in dependence is more pronounced, which suggests that the extraction and application of common factors can improve efficiency when explaining uncertainties of multiple stocks but also cause 

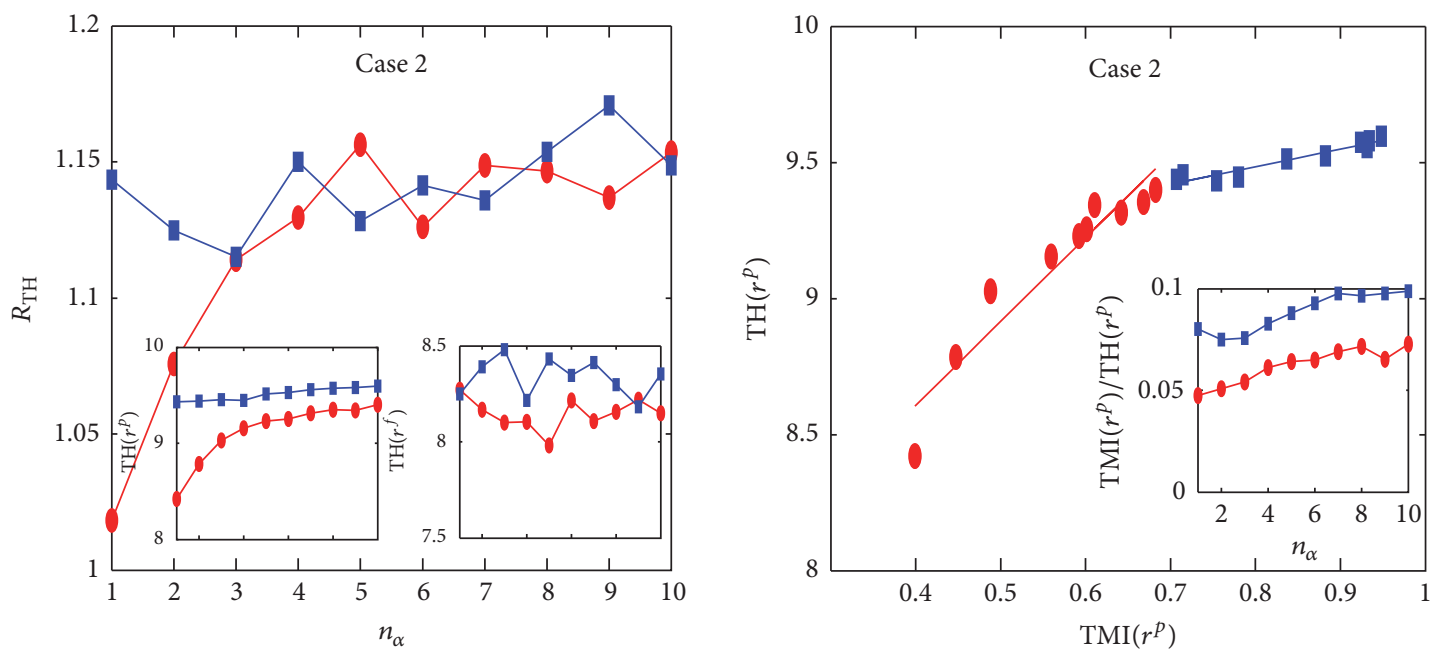

$\because n=2$

(1) $n=2$

- $n=4$

1 $n=4$
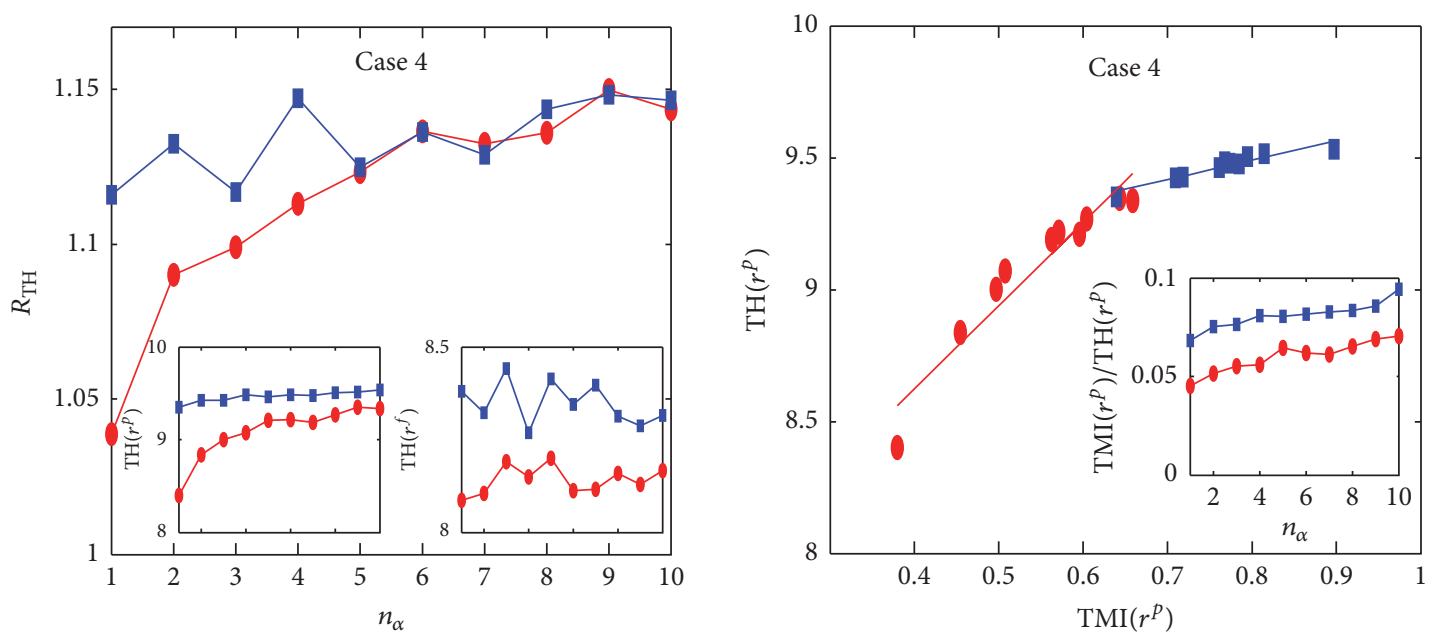

$-n=2$

- $n=4$
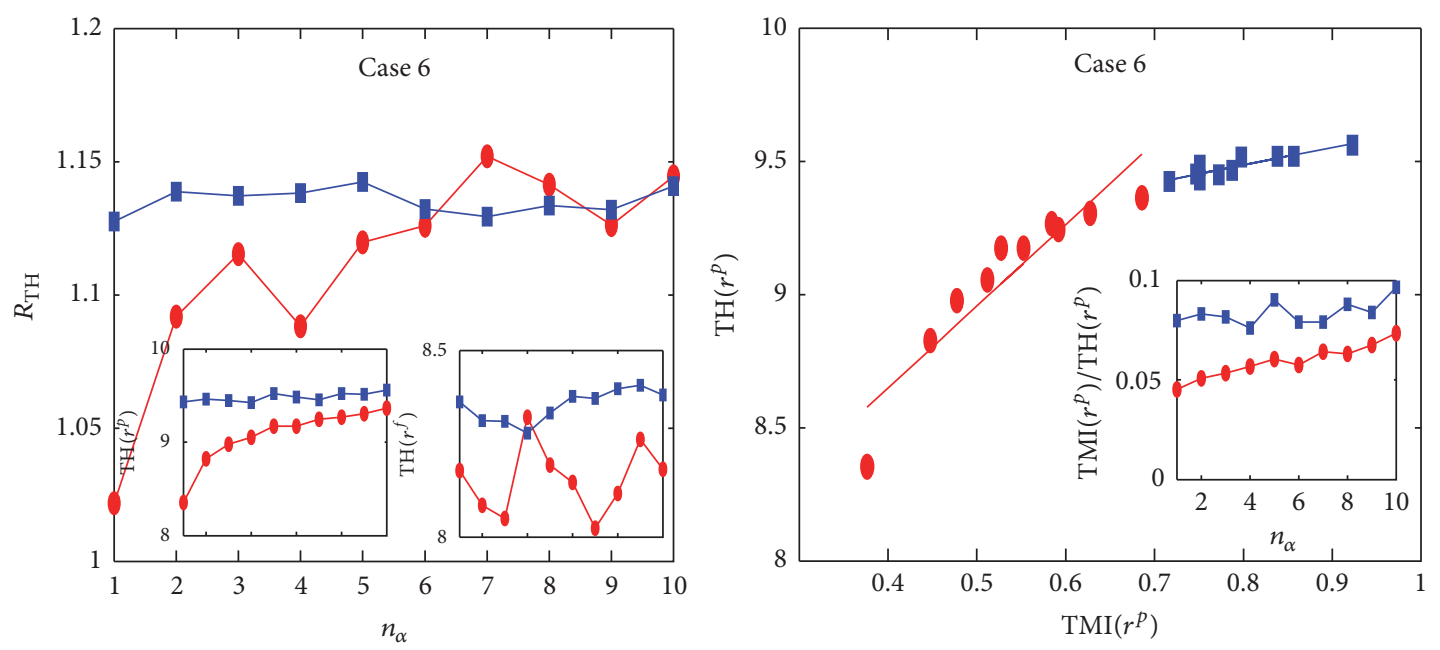

$\longrightarrow n=2$
$-n=4$

$n=2$

Figure 8: Continued. 

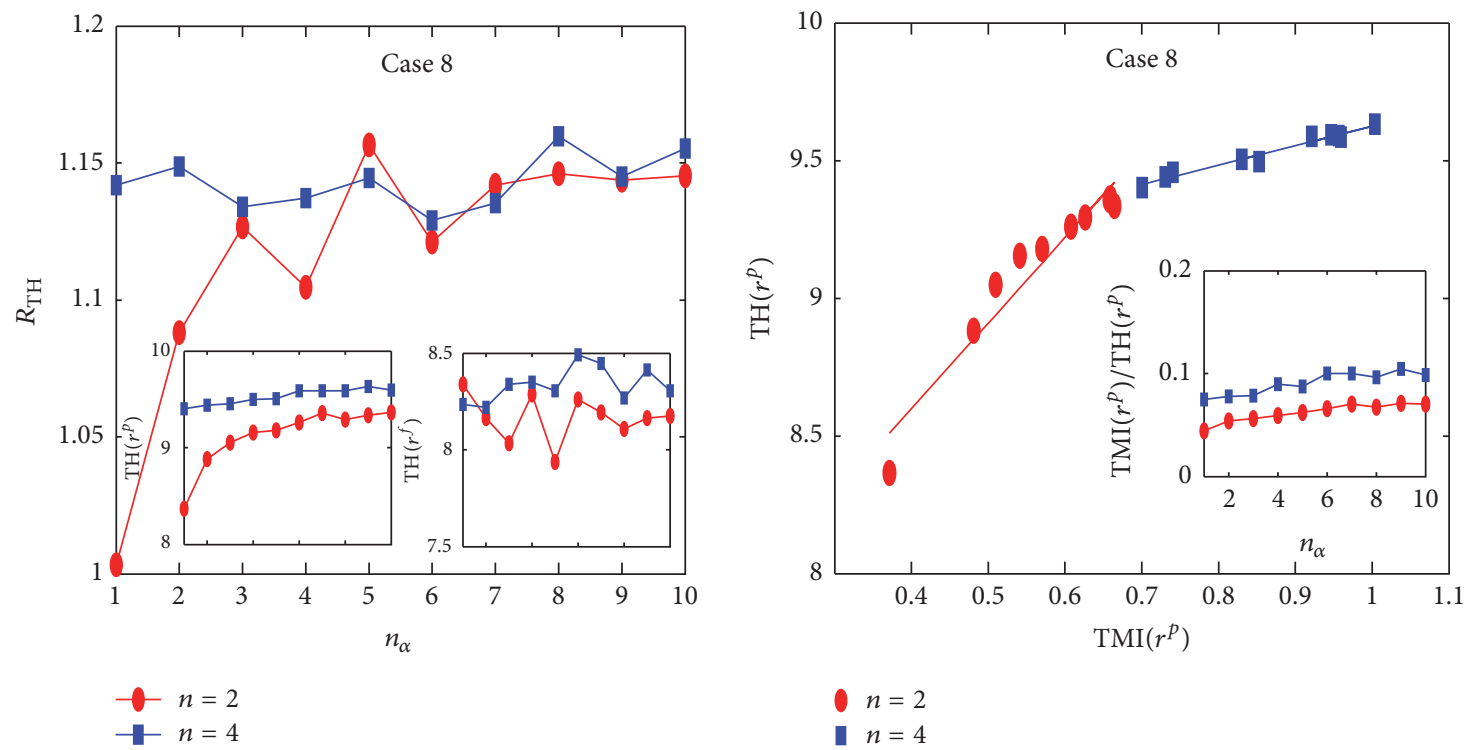

FIGURE 8: Results of robustness check of the model.

the dependence between them. This may undermine benefit of diversification brought by portfolio optimization. When taking into account the intrinsic values in our analysis, we find that the extraction and application of common factors are more effective in risk analysis and portfolio construction in the environment with more information sources and large information amount of common factors.

\section{Conflicts of Interest}

The authors declare that there are no conflicts of interest regarding the publication of this article.

\section{Acknowledgments}

This research is supported by National Natural Science Foundation of China Grant (nos. 71671037, 71371051, and 71201023), Humanities and Social Science Planning Foundation of the Ministry of Education of China (no. 16YJA630026), Social Science Fund Project of Jiangsu Province (no. 15GLC003), Fundamental Research Funds for the Central Universities, Funding of Jiangsu Innovation Program for Graduate Education (no. KYZZ16_0139), and Scientific Research Foundation of Graduate School of Southeast University (no. YBJJ1569).

\section{References}

[1] B. G. Malkiel and E. F. Fama, "Efficient capital markets: a review of theory and empirical work," The Journal of Finance, vol. 25, no. 2, pp. 383-417, 1970.

[2] E. F. Fama, "Market efficiency, long-term returns, and behavioral finance," Journal of Financial Economics, vol. 49, no. 3, pp. 283-306, 1998.

[3] W. F. de Bondt and R. Thaler, "Does the stock market overreact?", The Journal of Finance, vol. 40, no. 3, pp. 793-805, 1985.
[4] L. Fang and J. Peress, "Media coverage and the cross-section of stock returns," Journal of Finance, vol. 64, no. 5, pp. 2023-2052, 2009.

[5] W. Zhang, D. Shen, Y. Zhang, and X. Xiong, "Open source information, investor attention, and asset pricing," Economic Modelling, vol. 33, pp. 613-619, 2013.

[6] H. Che, X. Xiong, J. Yang, W. Zhang, and Y. Zhang, "How investor structure influences the yield, information dissemination efficiency, and liquidity," Discrete Dynamics in Nature and Society, vol. 2014, Article ID 742182, 2014.

[7] G. Moscarini, "Limited information capacity as a source of inertia," Journal of Economic Dynamics and control, vol. 28, no. 10, pp. 2003-2035, 2004.

[8] C. A. Sims, "Stickiness," Carnegie-rochester conference series on public policy, vol. 49, pp. 317-356, 1998.

[9] C. A. Sims, "Implications of rational inattention," Journal of Monetary Economics, vol. 50, no. 3, pp. 665-690, 2003.

[10] L. Peng and W. Xiong, "Investor attention, overconfidence and category learning," Journal of Financial Economics, vol. 80, no. 3, pp. 563-602, 2006.

[11] S. Van Nieuwerburgh and L. Veldkamp, "Information acquisition and under-diversification," Review of Economic Studies, vol. 77, no. 2, pp. 779-805, 2010.

[12] S. Van Nieuwerburgh and L. Veldkamp, "Information immobility and the home bias puzzle," Journal of Finance, vol. 64, no. 3, pp. 1187-1215, 2009.

[13] E. F. Fama and K. R. French, "Common risk factors in the returns on stocks and bonds," Journal of Financial Economics, vol. 33, no. 1, pp. 3-56, 1993.

[14] E. F. Fama and K. R. French, "Multifactor explanations of asset pricing anomalies," Journal of Finance, vol. 51, no. 1, pp. 55-84, 1996.

[15] C. Eom, G. Oh, and S. Kim, "Deterministic factors of stock networks based on cross-correlation in financial market," Physica A: Statistical Mechanics and its Applications, vol. 383, no. 1, pp. 139-146, 2007. 
[16] S. K. Mohanty, M. Nandha, A. Q. Turkistani, and M. Y. Alaitani, "Oil price movements and stock market returns: evidence from gulf cooperation council (GCC) countries," Global Finance Journal, vol. 22, no. 1, pp. 42-55, 2011.

[17] E. F. Fama and K. R. French, "Size, value, and momentum in international stock returns," Journal of Financial Economics, vol. 105, no. 3, pp. 457-472, 2012.

[18] G. H. Bauer and K. Vorkink, "Forecasting multivariate realized stock market volatility," Journal of Econometrics, vol. 160, no. 1, pp. 93-101, 2011.

[19] N. Jegadeesh and S. Titman, "Returns to buying winners and selling losers: implications for stock market efficiency," The Journal of Finance, vol. 48, no. 1, pp. 65-91, 1993.

[20] N. Jegadeesh, "Evidence of predictable behavior of security returns," The Journal of Finance, vol. 45, no. 3, pp. 881-898, 1990.

[21] Y. Amihud, "Illiquidity and stock returns: cross-section and time-series effects," Journal of Financial Markets, vol. 5, no. 1, pp. 31-56, 2002.

[22] S.-H. Chen and C.-H. Yeh, "Evolving traders and the business school with genetic programming: A new architecture of the agent-based artificial stock market," Journal of Economic Dynamics and Control, vol. 25, no. 3-4, pp. 363-393, 2001.

[23] S. Martinez-Jaramillo and E. P. K. Tsang, "An heterogeneous, endogenous and coevolutionary GP-based financial market," IEEE Transactions on Evolutionary Computation, vol. 13, no. 1, pp. 33-55, 2009.

[24] G. Bottazzi, G. Dosi, and I. Rebesco, "Institutional architectures and behavioral ecologies in the dynamics of financial markets," Journal of Mathematical Economics, vol. 41, no. 1-2, pp. 197-228, 2005.

[25] C. Chiarella and G. Iori, "A simulation analysis of the microstructure of double auction markets*," Quantitative Finance, vol. 2, no. 5, pp. 346-353, 2002.

[26] B. LeBaron, W. B. Arthur, and R. Palmer, "Time series properties of an artificial stock market," Journal of Economic Dynamics and Control, vol. 23, no. 9-10, pp. 1487-1516, 1999.

[27] R. G. Palmer, W. B. Arthur, J. H. Holland, and B. LeBaron, "An artificial stock market," Artificial Life and Robotics, vol. 3, no. 1, pp. 27-31, 1999.

[28] M. A. Bertella, F. R. Pires, L. Feng, and H. E. Stanley, "Confidence and the stock market: an agent-based approach," PLos One, vol. 9, no. 1, Article ID e83488, 2014.

[29] R. Yamamoto and H. Hirata, "Strategy switching in the Japanese stock market," Journal of Economic Dynamics and Control, vol. 37, no. 10, pp. 2010-2022, 2013.

[30] C. Chiarella, R. Dieci, and X.-Z. He, "Heterogeneous expectations and speculative behavior in a dynamic multi-asset framework," Journal of Economic Behavior and Organization, vol. 62, no. 3, pp. 408-427, 2007.

[31] L. E. Kodres and M. Pritsker, "A rational expectations model of financial contagion," Journal of Finance, vol. 57, no. 2, pp. 769799, 2002.

[32] C. Chiarella, G. Iori, and J. Perelló, "The impact of heterogeneous trading rules on the limit order book and order flows," Journal of Economic Dynamics and Control, vol. 33, no. 3, pp. 525-537, 2009.

[33] T. M. Cover and J. A. Thomas, Elements of Information Theory, John Wiley \& Sons, 2012.

[34] Y. Li, W. Zhang, Y. Zhang, X. Zhang, and X. Xiong, "Calibration of the agent-based continuous double auction stock market by scaling analysis," Information Sciences. An International Journal, vol. 256, pp. 46-56, 2014.
[35] W. Zhang, Z. Bi, and D. Shen, "Investor structure and the price-volume relationship in a continuous double auction market: an agent-based modeling perspective," Physica A: Statistical Mechanics and its Applications, vol. 467, pp. 345-355, 2017.

[36] C. E. Shannon, "A mathematical theory of communication," Bibliometrics, vol. 5, no. 1, pp. 3-55, 2001.

[37] D. A. Hsieth, "Chaos and nonlinear dynamics: application to financial markets," The Journal of Finance, vol. 46, no. 5, pp. 1839-1877, 1991.

[38] D. G. McMillan, "Nonlinear predictability of stock market returns: evidence from nonparametric and threshold models," International Review of Economics and Finance, vol. 10, no. 4, pp. 353-368, 2001.

[39] M. Beine, G. Capelle-Blancard, and H. Raymond, "International nonlinear causality between stock markets," European Journal of Finance, vol. 14, no. 8, pp. 663-686, 2008.

[40] P. Fiedor, "Networks in financial markets based on the mutual information rate," Physical Review E, vol. 89, no. 5, 2014.

[41] R. Menezes, A. Dionísio, and H. Hassani, "On the globalization of stock markets: an application of vector error correction model, mutual information and singular spectrum analysis to the G7 countries," Quarterly Review of Economics and Finance, vol. 52, no. 4, pp. 369-384, 2012.

[42] A. Dionisio, R. Menezes, and D. A. Mendes, "Mutual information: a measure of dependency for nonlinear time series," Physica A. Statistical Mechanics and its Applications, vol. 344, no. 1-2, pp. 326-329, 2004.

[43] M. Harré and T. Bossomaier, "Phase-transition-like behaviour of information measures in financial markets," EPL, vol. 87, no. 1, Article ID 18009, 2009.

[44] W. Shi and P. Shang, "Cross-sample entropy statistic as a measure of synchronism and cross-correlation of stock markets," Nonlinear Dynamics, vol. 71, no. 3, pp. 539-554, 2013. 


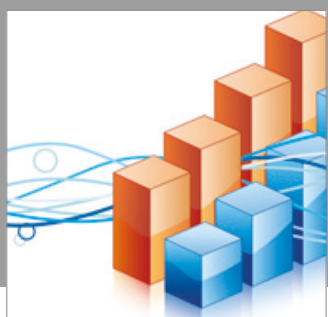

Advances in

Operations Research

vatersals

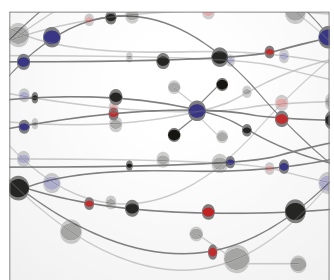

\section{The Scientific} World Journal
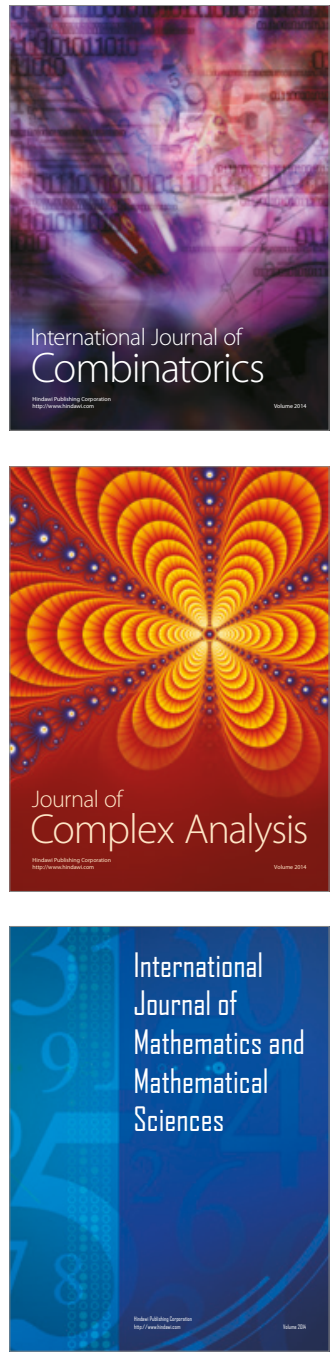
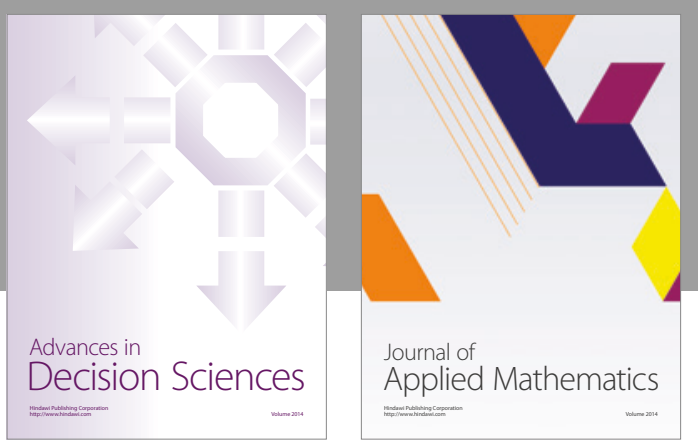

Algebra

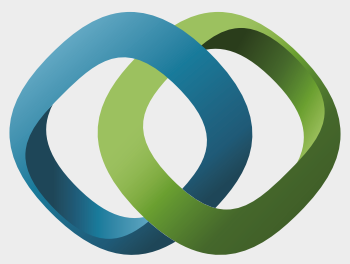

\section{Hindawi}

Submit your manuscripts at

https://www.hindawi.com
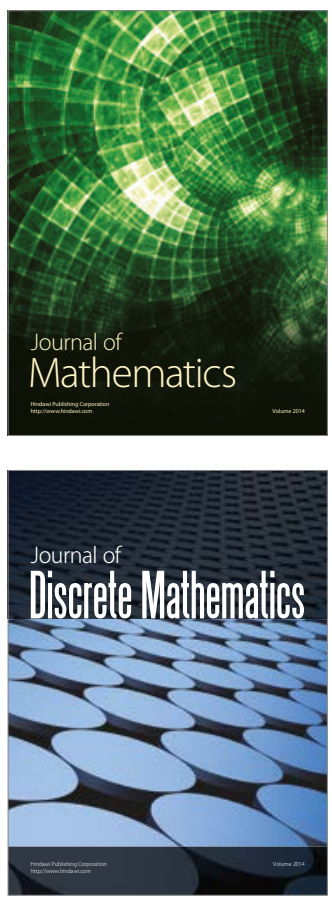

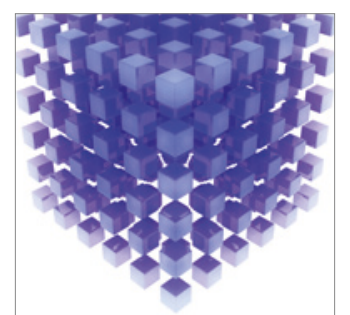

Mathematical Problems in Engineering
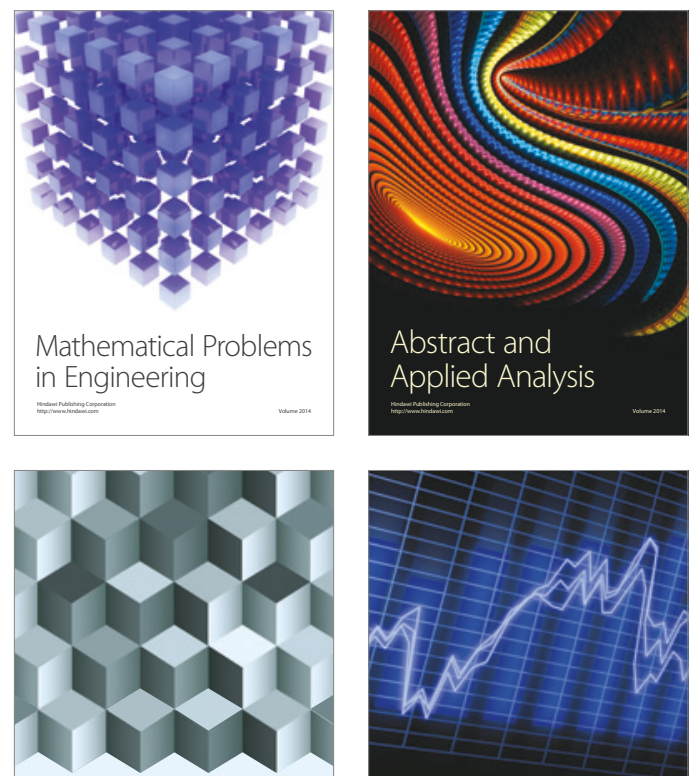

Journal of

Function Spaces

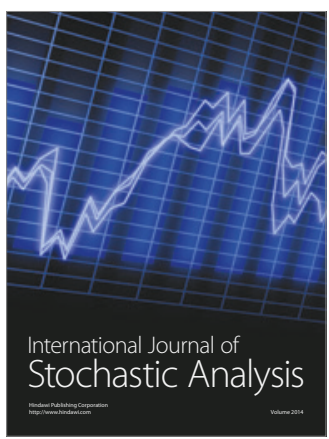

Probability and Statistics
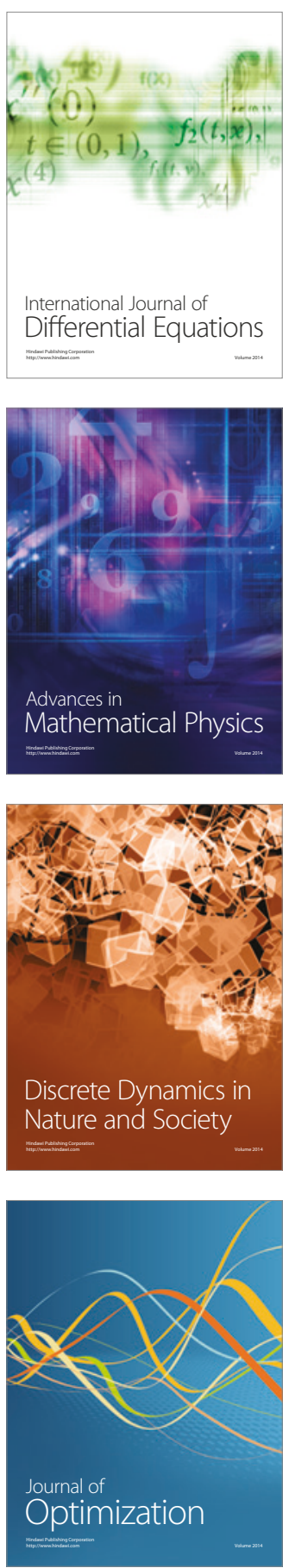